\title{
Turnover Liquidity and the Transmission of Monetary Policy
}

\author{
By Ricardo Lagos And SHENGXING Zhang*
}

\begin{abstract}
We provide empirical evidence of a novel liquidity-based transmission mechanism through which monetary policy influences asset markets, develop a model of this mechanism, and assess the ability of the quantitative theory to match the evidence. (JEL E44, E52, G12, G14, G35)
\end{abstract}

In most modern economies, central banks implement monetary policy indirectly, by intervening in certain financial markets (e.g., in the United States, the federal funds market and the market for treasury securities). The underlying idea is that the effects of those interventions on asset prices are transmitted to the rest of the economy to help achieve the ultimate policy objectives. Thus, the transmission mechanism of monetary policy to asset prices is important for understanding how monetary policy actually operates.

In this paper, we conduct an empirical, theoretical, and quantitative study of the effects of monetary policy on financial markets in general and the equity market in particular. We make three contributions. First, we provide original empirical evidence of a novel channel through which monetary policy influences financial markets: tight money increases the opportunity cost of holding the nominal assets used routinely to settle financial transactions (e.g., bank reserves, money balances), making these payment instruments scarcer. In turn, this scarcity reduces the resalability of financial assets, and this increased illiquidity leads to a reduction in price. We label this mechanism the turnover-liquidity (transmission) mechanism (of monetary policy). Second, to gain a deeper understanding of this mechanism, we develop a theory of trade in financial over-the-counter (OTC) markets (that nests the competitive benchmark as a special case) in which money is used as a medium of exchange in financial transactions. The model shows how the details of the market microstructure and

\footnotetext{
*Lagos: Department of Economics, New York University (email: ricardo.lagos@nyu.edu); Zhang: Department of Economics, London School of Economics (email: s.zhang31@1se.ac.uk). John Leahy was the coeditor for this article. This paper was submitted to the AER on January 9, 2017, and was accepted on December 17, 2018. Lagos is thankful for the support from the C.V. Starr Center for Applied Economics at NYU, and for the hospitality of Princeton University, University College London, the University of Minnesota, and the Federal Reserve Bank of Minneapolis. The views expressed herein are those of the authors and not necessarily those of the Federal Reserve Bank of Minneapolis or the Federal Reserve System. Zhang is thankful for the support from the Centre for Macroeconomics at LSE, the British Academy/Leverhulme Small Research Grant, and the ESRC-NSFC Grant "Building Debt Capital Markets in China." The authors declare that they have no relevant or material financial interests that relate to the research described in this paper.

${ }^{\dagger}$ Go to https://doi.org/10.1257/aer.20170045 to visit the article page for additional materials and author disclosure statements.
} 
the quantity of money shape the performance of financial markets (e.g., as gauged by standard measures of market liquidity), contribute to the determination of asset prices (e.g., through the resale option value of assets), and, consistent with the evidence we document, offer a liquidity-based explanation for the negative correlation between real stock returns and unexpected increases in the nominal interest rate that is used to implement monetary policy. Third, we bring the theory to the data. We calibrate a generalized version of the basic model and use it to conduct quantitative theoretical exercises designed to assess the ability of the theory to match the empirical effects of monetary policy on asset prices, both on policy announcement days and at longer horizons.

The rest of the paper is organized as follows. Section I presents the basic model. It considers a setting in which a financial asset that yields a dividend flow of consumption goods (e.g., an equity or a real bond) is demanded by investors who have time-varying heterogeneous valuations for the dividend. To achieve the gains from trade that arise from their heterogeneous valuations, investors participate in a bilateral market with random search that is intermediated by specialized dealers who have access to a competitive interdealer market. In the dealer-intermediated bilateral market, which has many of the stylized features of a typical OTC market structure but also nests the perfectly competitive market structure as a special case, investors and dealers seek to trade the financial asset using money as a means of payment. Periodically, dealers and investors are also able to rebalance their portfolios in a conventional Walrasian market. Equilibrium is characterized in Section II. Section III presents the main implications of the theory. Asset prices and conventional measures of financial liquidity (e.g., spreads, trade volume, and dealer supply of immediacy) are determined by the (real) quantity of money and the details of the microstructure where the asset trades (e.g., the degree of market power of dealers and the ease with which investors find counterparties). Generically, asset prices in the monetary economy exhibit a speculative premium whose size varies systematically with the market microstructure and the monetary policy stance. For example, a high anticipated opportunity cost of holding money reduces equilibrium real balances and distorts the asset allocation by causing too many assets to remain in the hands of investors with relatively low valuations, which depresses real asset prices.

Section IV is empirical. In it we revisit the finding, documented in previous empirical work, that surprise increases in the nominal policy rate cause sizable reductions in real stock returns on announcement days of the Federal Open Market Committee (FOMC). A 1 basis point unexpected increase in the policy rate causes a decrease of between 5 and 11 basis points in the stock market return on the day of the policy announcement. In addition, this section contains two new empirical findings. First, we document that episodes of unexpected policy tightening are also associated with large and persistent declines in stock turnover. Second, we find evidence that the magnitude of the reduction in return caused by the policy tightening is significantly larger for stocks that are normally traded more actively, e.g., stocks with higher turnover rates. For example, in response to an unexpected increase in the policy rate, the announcement-day decline in the return of a stock in the ninety-fifth percentile of turnover rates is about 2.5 times larger than that of a stock in the fifth percentile. The empirical evidence in this section suggests a mechanism whereby monetary policy affects asset prices through a reduction in turnover liquidity. 
In Section $\mathrm{V}$ we formulate, calibrate, and simulate a generalized version of the basic model and use it to assess the ability of the theory to fit the empirical evidence on the effects of monetary shocks on aggregate stock returns as well as the new cross-sectional evidence on the turnover-liquidity transmission mechanism. Section VI concludes. Online Appendix Section A contains all proofs. Online Appendix Sections B, C, D, and E contain supplementary material. Online Appendix Section B covers technical aspects of the data, estimation, and simulation. Online Appendix Section C contains additional theoretical derivations and results. Online Appendix Section D verifies the robustness of the empirical and quantitative findings. This paper is related to four areas of research: search-theoretic models of money, search-theoretic models of financial trade in OTC markets, resale option theories of asset price bubbles, and an extensive empirical literature that studies the effects of monetary policy on asset prices. Online Appendix Section E places our contribution in the context of all these literatures.

\section{Model}

Time is represented by a sequence of periods indexed by $t=0,1, \ldots$ Each period is divided into two subperiods where different activities take place. There is a continuum of infinitely lived agents called investors, each identified with a point in the set $\mathcal{I}=[0,1]$. There is also a continuum of infinitely lived agents called dealers, each identified with a point in the $\operatorname{set} \mathcal{D}=[0,1]$. All agents discount payoffs across periods with the discount factor $\beta \equiv 1 /(1+r)$, where $r>0$ denotes the real interest rate. In every period, there is a continuum of active production units with measure $A^{s} \in \mathbb{R}_{++}$. Every active unit yields an exogenous dividend $y_{t} \in \mathbb{R}_{+}$ of a perishable consumption good at the end of the first subperiod of period $t$. (Each active unit yields the same dividend as every other active unit, so $y_{t} A^{s}$ is the aggregate dividend.) At the beginning of every period, every active unit is subject to an independent idiosyncratic shock that renders it permanently unproductive with probability $1-\delta \in[0,1)$. If a production unit remains active, its dividend in period $t$ is $y_{t}=\gamma_{t} y_{t-1}$ where $\gamma_{t}$ is a nonnegative random variable with cumulative distribution function $\Gamma$, i.e., $\operatorname{Pr}\left(\gamma_{t} \leq \gamma\right)=\Gamma(\gamma)$, and mean $\bar{\gamma} \in\left(0,(\beta \delta)^{-1}\right)$. The time $t$ dividend becomes known to all agents at the beginning of period $t$, and at that time each failed production unit is replaced by a new unit that yields dividend $y_{t}$ in the initial period and follows the same stochastic process as other active units thereafter (the dividend of the initial set of production units, $y_{0} \in \mathbb{R}_{++}$, is given at $t=0$ ). In the second subperiod of every period, every agent has access to a linear production technology that transforms effort into a perishable consumption good.

For each active production unit, there is a durable and perfectly divisible equity share that represents the bearer's ownership of the production unit and confers him the right to collect dividends. At the beginning of every period $t \geq 1$, each investor receives an endowment of $(1-\delta) A^{s}$ equity shares corresponding to the new production units. (When a production unit fails, its equity share disappears.) There is a second financial instrument, money, that is intrinsically useless (it is not an argument of any utility or production function, and unlike equity, ownership of money does not constitute a right to collect any resources). The stock of money at time $t$ is denoted $A_{t}^{m}$. The initial stock of money, $A_{0}^{m} \in \mathbb{R}_{++}$, is given and $A_{t+1}^{m}=\mu A_{t}^{m}$, 
with $\mu \in \mathbb{R}_{++}$. A monetary authority injects or withdraws money via lump-sum transfers or taxes to investors in the second subperiod of every period. At the beginning of period $t=0$, each investor is endowed with a portfolio of equity shares and money. All financial instruments are perfectly recognizable, cannot be forged, and can be traded in every subperiod.

In the second subperiod of every period, all agents can trade the consumption good produced in that subperiod, equity shares, and money in a spot Walrasian market. In the first subperiod of every period, trading is organized as follows. Investors can trade equity shares and money in a random bilateral OTC market with dealers, while dealers can also trade equity shares and money with other dealers in a spot Walrasian dealer market. We use $\alpha \in[0,1]$ to denote the probability that an individual investor is able to make contact with a dealer in the OTC market. (The probability that a dealer contacts an investor is also $\alpha$.) Once a dealer and an investor have contacted each other, the pair negotiates the quantity of equity shares and money that the dealer will trade in the dealer market on behalf of the investor and a fee for the dealer's intermediation services. We assume the terms of the trade between an investor and a dealer in the OTC market are determined by Nash bargaining where $\theta \in[0,1]$ is the investor's bargaining power. The timing is that the round of OTC trade takes place in the first subperiod and ends before production units yield dividends. Hence, equity is traded cum dividend in the OTC market (and in the dealer market) of the first subperiod and ex dividend in the Walrasian market of the second subperiod. ${ }^{1}$ Asset purchases in the OTC market cannot be financed by borrowing (e.g., due to anonymity and lack of commitment and enforcement). This assumption and the structure of preferences described below create the need for a medium of exchange in the OTC market.

An individual dealer's preferences are represented by

$$
E_{0}^{d} \sum_{t=0}^{\infty} \beta^{t}\left(c_{d t}-h_{d t}\right),
$$

where $c_{d t}$ is his consumption of the homogeneous good that is produced, traded, and consumed in the second subperiod of period $t$, and $h_{d t}$ is the utility cost from exerting $h_{d t}$ units of effort to produce this good. The expectation operator $E_{0}^{d}$ is with respect to the probability measure induced by the dividend process and the random trading process in the OTC market. Dealers get no utility from the dividend good. ${ }^{2}$ An individual investor's preferences are represented by

$$
E_{0} \sum_{t=0}^{\infty} \beta^{t}\left(\varepsilon_{i t} y_{i t}+c_{i t}-h_{i t}\right),
$$

where $y_{i t}$ is the quantity of the dividend good that investor $i$ consumes at the end of the first subperiod of period $t, c_{i t}$ is his consumption of the homogeneous good that is produced, traded, and consumed in the second subperiod of period $t$, and $h_{i t}$ is

\footnotetext{
${ }^{1}$ As in previous search models of OTC markets (e.g., see Duffie, Gârleanu, and Pedersen 2005 and Lagos and Rocheteau 2009), an investor must own the equity share in order to consume the dividend.

${ }^{2}$ This assumption implies that dealers have no direct consumption motive for holding the equity share. It is easy to relax, but we adopt it because it is the standard benchmark in the search-based OTC literature, e.g., see Duffie, Gârleanu, and Pedersen (2005); Lagos and Rocheteau (2009); Lagos, Rocheteau, and Weill (2011); and Weill (2007).
} 
the utility cost from exerting $h_{i t}$ units of effort to produce this good. The variable $\varepsilon_{i t}$ denotes the realization of a valuation shock that is distributed independently over time and across agents, with a differentiable cumulative distribution function $G$ on the support $\left[\varepsilon_{L}, \varepsilon_{H}\right] \subseteq[0, \infty]$, and $\bar{\varepsilon}=\int \varepsilon d G(\varepsilon)$. Investor $i$ learns his realization $\varepsilon_{i t}$ at the beginning of period $t$, before the OTC trading round. The expectation operator $E_{0}$ is with respect to the probability measure induced by the dividend process, the investor's valuation shock, and the random trading process in the OTC market. ${ }^{3}$

Consider a social planner who wishes to maximize the sum of all agents' expected discounted utilities subject to the same meeting frictions that agents face in the decentralized formulation. Specifically, in the first subperiod of every period, the planner can only reallocate assets among all dealers and the measure $\alpha$ of investors who contact dealers at random. In online Appendix Section C (Proposition 11), we prove the allocation that solves the planner's problem is characterized by the following two properties: (i) only dealers carry equity between periods, and (ii) dealers do not carry assets after the OTC round of trade, and among those investors who have a trading opportunity with a dealer in the OTC market, only those with the highest valuation hold equity shares at the end of the first subperiod. The planner's problem is useful to highlight the two (re)allocation motives that will drive equilibrium outcomes in the following section. ${ }^{4}$ First, the planner implements an efficient allocation of assets in the OTC trading round: since an investor's utility from the dividend good is linear, and dealers get no utility from the dividend good, the efficient allocation requires that all the assets held by dealers at the beginning of the period are reallocated to the set of investors with the highest valuation for the dividend. Second, by allocating all assets to dealers at the end of every period, the planner ensures that all assets can be reallocated to the set of highest valuation investors in the following OTC trading round with probability 1 (notice that if instead a set of investors were to enter the OTC round holding the asset, most of these investors would draw valuation shocks strictly lower than $\varepsilon_{H}$, and the planner would only be able to reallocate a fraction $\alpha<1$ of those assets to the highest valuation investors who contacted dealers).

\section{Equilibrium}

Consider the determination of the terms of trade in a bilateral meeting in the OTC round of period $t$ between a dealer with portfolio $\mathbf{a}_{d t}$ and an investor with portfolio $\mathbf{a}_{i t}$ and valuation $\varepsilon$. Let $\overline{\mathbf{a}}_{t}=\left(\bar{a}_{t}^{m}, \bar{a}_{t}^{s}\right)$ denote the investor's post-trade portfolio and let $\varphi_{t}$ denote the fee the dealer charges for his intermediation services. The fee is expressed in terms of the second-subperiod consumption good and paid by the investor in the second subperiod. $\left[\right.$ We assume $\left(\overline{\mathbf{a}}_{t}, \varphi_{t}\right)$ is determined

\footnotetext{
${ }^{3}$ The valuation shock stands in for the various idiosyncratic reasons why individual investors may wish to hold different quantities of a certain asset at different points in time, such as differences in their liquidity needs, financing or financial-distress costs, or hedging needs (e.g., correlation of asset returns with endowments). Several papers that build on the work of Duffie, Gârleanu, and Pedersen (2005) have formalized the "hedging needs" interpretation. Examples include Duffie, Gârleanu, and Pedersen (2007); Gârleanu (2009); and Vayanos and Weill (2008).

${ }^{4}$ Below we show that, under the Friedman rule, the decentralized monetary equilibrium implements the planner's allocation (see Corollary 1).

${ }^{5}$ In the working paper version of this model (Lagos and Zhang 2015), we instead assume that the investor must pay the intermediation fee on the spot, i.e., with money or equity. The alternative formulation we use here makes the analysis and the exposition much simpler while the main economic mechanisms are essentially unchanged.
} 
by the Nash bargaining solution where the investor has bargaining power $\theta \in[0,1]$. Let $\hat{W}_{t}^{D}\left(\mathbf{a}_{d t}, \varphi_{t}\right)$ denote the maximum expected discounted payoff of a dealer with portfolio $\mathbf{a}_{d t}$ and earned fee $\varphi_{t}$ when he reallocates his portfolio in the dealer market of period $t$. Let $W_{t}^{I}\left(\mathbf{a}_{i t}, \varphi_{t}\right)$ denote the maximum expected discounted payoff at the beginning of the second subperiod of period $t$ (after the production units have borne dividends) of an investor who is holding portfolio $\mathbf{a}_{i t}$ and has to pay a fee $\varphi_{t}$. For each $t$, define a pair of functions $\bar{a}_{t}^{k}: \mathbb{R}_{+}^{2} \times\left[\varepsilon_{L}, \varepsilon_{H}\right] \rightarrow \mathbb{R}_{+}$for $k=m, s$ and a function $\varphi_{t}: \mathbb{R}_{+}^{2} \times\left[\varepsilon_{L}, \varepsilon_{H}\right] \rightarrow \mathbb{R}$, and let $\overline{\mathbf{a}}_{t}\left(\mathbf{a}_{i t}, \varepsilon\right)=\left(\bar{a}_{t}^{m}\left(\mathbf{a}_{i t}, \varepsilon\right), \bar{a}_{t}^{s}\left(\mathbf{a}_{i t}, \varepsilon\right)\right)$ for each $\left(\mathbf{a}_{i t}, \varepsilon\right) \in \mathbb{R}_{+}^{2} \times\left[\varepsilon_{L}, \varepsilon_{H}\right]$. We use $\left[\overline{\mathbf{a}}_{t}\left(\mathbf{a}_{i t}, \varepsilon\right), \varphi_{t}\left(\mathbf{a}_{i t}, \varepsilon\right)\right]$ to represent the bargaining outcome for a bilateral meeting at time $t$ between an investor with portfolio $\mathbf{a}_{i t}$ and valuation $\varepsilon$, and a dealer with portfolio $\mathbf{a}_{d t}$. That is, $\left[\overline{\mathbf{a}}_{t}\left(\mathbf{a}_{i t}, \varepsilon\right), \varphi_{t}\left(\mathbf{a}_{i t}, \varepsilon\right)\right]$ solves

$$
\begin{aligned}
\max _{\left(\overline{\mathbf{a}}_{t}, \varphi_{t}\right) \in \mathbb{R}_{+}^{2} \times \mathbb{R}}\left\{\left[\varepsilon y_{t} \bar{a}_{t}^{s}+\right.\right. & \left.W_{t}^{I}\left(\overline{\mathbf{a}}_{t}, \varphi_{t}\right)-\varepsilon y_{t} a_{i t}^{s}-W_{t}^{I}\left(\mathbf{a}_{i t}, 0\right)\right]^{\theta} \\
& \left.\times\left[\hat{W}_{t}^{D}\left(\mathbf{a}_{d t}, \varphi_{t}\right)-\hat{W}_{t}^{D}\left(\mathbf{a}_{d t}, 0\right)\right]^{1-\theta}\right\},
\end{aligned}
$$

subject to

$$
\begin{aligned}
\bar{a}_{t}^{m}+p_{t} \bar{a}_{t}^{s} & \leq a_{i t}^{m}+p_{t} a_{i t}^{s}, \\
\hat{W}_{t}^{D}\left(\mathbf{a}_{d t}, 0\right) & \leq \widehat{W}_{t}^{D}\left(\mathbf{a}_{d t}, \varphi_{t}\right), \\
\varepsilon y_{t} a_{i t}^{s}+W_{t}^{I}\left(\mathbf{a}_{i t}, 0\right) & \leq \varepsilon y_{t} \bar{a}_{t}^{s}+W_{t}^{I}\left(\overline{\mathbf{a}}_{t}, \varphi_{t}\right),
\end{aligned}
$$

where $p_{t}$ is the dollar price of an equity share in the dealer market of period $t$.

Let $W_{t}^{D}\left(\mathbf{a}_{t}, \varphi_{t}\right)$ denote the maximum expected discounted payoff of a dealer who has earned fee $\varphi_{t}$ in the OTC round of period $t$ and, at the beginning of the second subperiod of period $t$, is holding portfolio $\mathbf{a}_{t}$. Then the dealer's value of trading in the dealer market is

$$
\widehat{W}_{t}^{D}\left(\mathbf{a}_{t}, \varphi_{t}\right)=\max _{\hat{\mathbf{a}}_{t} \in \mathbb{R}_{+}^{2}} W_{t}^{D}\left(\hat{\mathbf{a}}_{t}, \varphi_{t}\right)
$$

subject to

$$
\hat{a}_{t}^{m}+p_{t} \hat{a}_{t}^{s} \leq a_{t}^{m}+p_{t} a_{t}^{s}
$$

where $\hat{\mathbf{a}}_{t} \equiv\left(\hat{a}_{t}^{m}, \hat{a}_{t}^{s}\right)$. For each $t$, define a pair of functions, $\hat{a}_{t}^{k}: \mathbb{R}_{+}^{2} \rightarrow \mathbb{R}_{+}$ for $k=m, s$, and let $\hat{\mathbf{a}}_{t}\left(\mathbf{a}_{t}\right)=\left(\hat{a}_{t}^{m}\left(\mathbf{a}_{t}\right), \hat{a}_{t}^{s}\left(\mathbf{a}_{t}\right)\right)$ denote the solution to (2).

Let $V_{t}^{D}\left(\mathbf{a}_{t}\right)$ denote the maximum expected discounted payoff of a dealer who enters the OTC round of period $t$ with portfolio $\mathbf{a}_{t} \equiv\left(a_{t}^{m}, a_{t}^{s}\right)$. Let $\phi_{t} \equiv\left(\phi_{t}^{m}, \phi_{t}^{s}\right)$, where $\phi_{t}^{m}$ is the real price of money and $\phi_{t}^{s}$ the real ex dividend price of equity in the second subperiod of period $t$ (both expressed in terms of the second subperiod consumption good). Then,

$$
W_{t}^{D}\left(\mathbf{a}_{t}, \varphi_{t}\right)=\max _{\left(c_{t}, h_{t}, \tilde{\mathbf{a}}_{t+1}\right) \in \mathbb{R}_{+}^{4}}\left[c_{t}-h_{t}+\beta E_{t} V_{t+1}^{D}\left(\mathbf{a}_{t+1}\right)\right]
$$


subject to

$$
c_{t}+\phi_{t} \tilde{\mathbf{a}}_{t+1} \leq h_{t}+\varphi_{t}+\phi_{t} \mathbf{a}_{t}
$$

where $\tilde{\mathbf{a}}_{t+1} \equiv\left(\tilde{a}_{t+1}^{m}, \tilde{a}_{t+1}^{s}\right), \mathbf{a}_{t+1}=\left(\tilde{a}_{t+1}^{m}, \delta \tilde{a}_{t+1}^{s}\right), E_{t}$ is the conditional expectation over the next-period realization of the dividend, and $\phi_{t} \mathbf{a}_{t}$ denotes the dot product of $\phi_{t}$ and $\mathbf{a}_{t}$. Similarly, let $V_{t}^{I}\left(\mathbf{a}_{t}, \varepsilon\right)$ denote the maximum expected discounted payoff of an investor with valuation $\varepsilon$ and portfolio $\mathbf{a}_{t} \equiv\left(a_{t}^{m}, a_{t}^{s}\right)$ at the beginning of the OTC round of period $t$. Then,

$$
W_{t}^{I}\left(\mathbf{a}_{t}, \varphi_{t}\right)=\max _{\left(c_{t}, h_{t}, \tilde{\mathbf{a}}_{t+1}\right) \in \mathbb{R}_{+}^{4}}\left[c_{t}-h_{t}+\beta E_{t} \int V_{t+1}^{I}\left(\mathbf{a}_{t+1}, \varepsilon^{\prime}\right) d G\left(\varepsilon^{\prime}\right)\right],
$$

subject to

$$
c_{t}+\phi_{t} \tilde{\mathbf{a}}_{t+1} \leq h_{t}-\varphi_{t}+\phi_{t} \mathbf{a}_{t}+T_{t}
$$

where $\mathbf{a}_{t+1}=\left(\tilde{a}_{t+1}^{m}, \delta \tilde{a}_{t+1}^{s}+(1-\delta) A^{s}\right)$ and $T_{t} \in \mathbb{R}$ is the real value of the time $t$ lump-sum monetary transfer. ${ }^{6}$

The value function of an investor who enters the OTC round of period $t$ with portfolio $\mathbf{a}_{t}$ and valuation $\varepsilon$ is

$$
\begin{aligned}
V_{t}^{I}\left(\mathbf{a}_{t}, \varepsilon\right)= & \alpha\left\{\varepsilon y_{t} \bar{a}_{t}^{s}\left(\mathbf{a}_{t}, \varepsilon\right)+W_{t}^{I}\left[\overline{\mathbf{a}}_{t}\left(\mathbf{a}_{t}, \varepsilon\right), \varphi_{t}\left(\mathbf{a}_{t}, \varepsilon\right)\right]\right\} \\
& +(1-\alpha)\left[\varepsilon y_{t} a_{t}^{s}+W_{t}^{I}\left(\mathbf{a}_{t}, 0\right)\right] .
\end{aligned}
$$

The value function of a dealer who enters the OTC round of period $t$ with portfolio $\mathbf{a}_{t}$ is

$$
V_{t}^{D}\left(\mathbf{a}_{t}\right)=\alpha \int \hat{W}_{t}^{D}\left[\mathbf{a}_{t}, \varphi_{t}\left(\mathbf{a}_{i t}, \varepsilon\right)\right] d H_{I t}\left(\mathbf{a}_{i t}, \varepsilon\right)+(1-\alpha) \hat{W}_{t}^{D}\left(\mathbf{a}_{t}, 0\right),
$$

where $H_{I t}$ is the cumulative distribution function over the portfolios and valuations of the investors the dealer may contact in the OTC market of period $t$.

Let $j \in\{D, I\}$ denote the agent type, i.e., $D$ for dealers and $I$ for investors. Then for $j \in\{D, I\}$, let $A_{j t}^{m}$ and $A_{j t}^{s}$ denote the quantities of money and equity shares, respectively, held by all agents of type $j$ at the beginning of the OTC round of period $t$ (after production units have depreciated and been replaced). That is, $A_{j t}^{m}=\int a_{t}^{m} d F_{j t}\left(\mathbf{a}_{t}\right)$ and $A_{j t}^{s}=\int a_{t}^{s} d F_{j t}\left(\mathbf{a}_{t}\right)$, where $F_{j t}$ is the cumulative distribution function over portfolios $\mathbf{a}_{t}=\left(a_{t}^{m}, a_{t}^{s}\right)$ held by agents of type $j$ at the beginning of the OTC round of period $t$. Let $\tilde{A}_{j t+1}^{m}$ and $\tilde{A}_{j t+1}^{s}$ denote the total quantities of money and shares held by all agents of type $j$ at the end of period $t$, i.e., $\tilde{A}_{D t+1}^{k}=\int_{\mathcal{D}} \tilde{a}_{j t+1}^{k} d j$ and $\tilde{A}_{I t+1}^{k}=\int_{\mathcal{I}} \tilde{a}_{i t+1}^{k} d i$ for $k \in\{s, m\}$, with $A_{D t+1}^{m}=\tilde{A}_{D t+1}^{m}, \quad A_{D t+1}^{s}=\delta \tilde{A}_{D t+1}^{s}, \quad A_{I t+1}^{m}=\tilde{A}_{I t+1}^{m}, \quad$ and

\footnotetext{
${ }^{6}$ In terms of notation, our convention is to use overbars, e.g., $\mathbf{a}$, for an investor's post-trade portfolio at the end of the first subperiod; overtildes, e.g., ã for the portfolio an investor chooses at the end of the second subperiod; and hats, e.g., â, for the post-trade portfolio a dealer chooses at the end of the first subperiod (after the round of trade with investors has taken place).
} 
$A_{I t+1}^{s}=\delta \tilde{A}_{I t+1}^{s}+(1-\delta) A^{s}$. Let $\bar{A}_{D t}^{m}$ and $\bar{A}_{D t}^{s}$ denote the quantities of money and shares held after the OTC round of trade of period $t$ by all the dealers, and let $\bar{A}_{I t}^{m}$ and $\bar{A}_{I t}^{s}$ denote the quantities of money and shares held after the OTC round of trade of period $t$ by all the investors who are able to trade in the first subperiod. For asset $k \in\{s, m\}, \bar{A}_{D t}^{k}=\int \hat{a}_{t}^{k}\left(\mathbf{a}_{t}\right) d F_{D t}\left(\mathbf{a}_{t}\right)$ and $\bar{A}_{I t}^{k}=\alpha \int \bar{a}_{t}^{k}\left(\mathbf{a}_{t}, \varepsilon\right) d H_{I t}\left(\mathbf{a}_{t}, \varepsilon\right)$. We are now ready to define an equilibrium.

DEFINITION 1: An equilibrium is a sequence of prices, $\left\{1 / p_{t}, \phi_{t}^{m}, \phi_{t}^{s}\right\}_{t=0}^{\infty}$, bilateral terms of trade in the OTC market, $\left\{\overline{\mathbf{a}}_{t}, \varphi_{t}\right\}_{t=0}^{\infty}$, dealer portfolios, $\left\{\left\langle\hat{\mathbf{a}}_{d t}, \tilde{\mathbf{a}}_{d t+1}, \mathbf{a}_{d t+1}\right\rangle_{d \in \mathcal{D}}\right\}_{t=0}^{\infty}$, and investor portfolios, $\left\{\left\langle\tilde{\mathbf{a}}_{i t+1}, \mathbf{a}_{i t+1}\right\rangle_{i \in \mathcal{I}}\right\}_{t=0}^{\infty}$, such that for all $t:(i)$ the bilateral terms of trade $\left\{\overline{\mathbf{a}}_{t}, \varphi_{t}\right\}_{t=0}^{\infty}$ solve (1), (ii) taking prices and the bargaining protocol as given, the portfolios $\left\langle\hat{\mathbf{a}}_{d t}, \tilde{\mathbf{a}}_{d t+1}, \mathbf{a}_{d t+1}\right\rangle$ solve the individual dealer's problems (2) and (3), and the portfolios $\left\langle\tilde{\mathbf{a}}_{i t+1}, \mathbf{a}_{i t+1}\right\rangle$ solve the individual investor's problem (4), and (iii) prices, $\left\{1 / p_{t}, \phi_{t}^{m}, \phi_{t}^{s}\right\}_{t=0}^{\infty}$, are such that all Walrasian markets clear, i.e., $\tilde{A}_{D t+1}^{s}+\tilde{A}_{I t+1}^{s}=A^{s}$ (the end-of-period $t$ Walrasian market for equity clears), $\tilde{A}_{D t+1}^{m}+\tilde{A}_{I t+1}^{m}=A_{t+1}^{m}$ (the end-of-period $t$ Walrasian market for money clears), and $\bar{A}_{D t}^{k}+\bar{A}_{I t}^{k}=A_{D t}^{k}+\alpha A_{I t}^{k}$ for $k=s, m$ (the period $t$ OTC dealer markets for equity and money clear). An equilibrium is "monetary" if $\phi_{t}^{m}>0$ for all t and "nonmonetary" otherwise.

The following result characterizes the equilibrium post-trade portfolios of dealers and investors in the OTC market, taking beginning-of-period portfolios as given.

LEMMA 1: Define $\varepsilon_{t}^{*} \equiv \frac{p_{t} \phi_{t}^{m}-\phi_{t}^{s}}{y_{t}}$ and

$$
\chi\left(\varepsilon_{t}^{*}, \varepsilon\right) \begin{cases}=1 & \text { if } \varepsilon_{t}^{*}<\varepsilon \\ \in[0,1] & \text { if } \varepsilon_{t}^{*}=\varepsilon \\ =0 & \text { if } \varepsilon<\varepsilon_{t}^{*} .\end{cases}
$$

Consider a bilateral meeting in the OTC round of period $t$ between a dealer and an investor with portfolio $\mathbf{a}_{t}$ and valuation $\varepsilon$. The investor's post-trade portfolio, $\left[\bar{a}_{t}^{m}\left(\mathbf{a}_{t}, \varepsilon\right), \bar{a}_{t}^{s}\left(\mathbf{a}_{t}, \varepsilon\right)\right]$, is given by

$$
\begin{aligned}
\bar{a}_{t}^{m}\left(\mathbf{a}_{t}, \varepsilon\right) & =\left[1-\chi\left(\varepsilon_{t}^{*}, \varepsilon\right)\right]\left(a_{t}^{m}+p_{t} a_{t}^{s}\right), \\
\bar{a}_{t}^{s}\left(\mathbf{a}_{t}, \varepsilon\right) & =\chi\left(\varepsilon_{t}^{*}, \varepsilon\right)\left(1 / p_{t}\right)\left(a_{t}^{m}+p_{t} a_{t}^{s}\right),
\end{aligned}
$$

and the intermediation fee charged by the dealer is

$$
\varphi_{t}\left(\mathbf{a}_{t}, \varepsilon\right)=(1-\theta)\left(\varepsilon-\varepsilon_{t}^{*}\right)\left[\chi\left(\varepsilon_{t}^{*}, \varepsilon\right) \frac{1}{p_{t}} a_{t}^{m}-\left[1-\chi\left(\varepsilon_{t}^{*}, \varepsilon\right)\right] a_{t}^{s}\right] y_{t} .
$$

A dealer who enters the OTC market with portfolio $\mathbf{a}_{d t}$ exits the OTC market with portfolio $\left[\hat{a}_{t}^{m}\left(\mathbf{a}_{d t}\right), \hat{a}_{t}^{s}\left(\mathbf{a}_{d t}\right)\right]=\left[\bar{a}_{t}^{m}\left(\mathbf{a}_{d t}, 0\right), \bar{a}_{t}^{s}\left(\mathbf{a}_{d t}, 0\right)\right]$.

Lemma 1 offers a full characterization of the post-trade portfolios of investors and dealers in the OTC market. First, the bargaining outcome depends on whether 
the investor's valuation, $\varepsilon$, is above or below a cutoff, $\varepsilon_{t}^{*}$. If $\varepsilon_{t}^{*}<\varepsilon$, the investor uses all his cash to buy equity. If $\varepsilon<\varepsilon_{t}^{*}$, he sells all his equity holding for cash. The intermediation fee earned by the dealer is equal to a share $1-\theta$ of the investor's gain from trade. The dealer's post-trade portfolio is the same as that of an investor with $\varepsilon=0$.

We focus the analysis on recursive equilibria, that is, equilibria in which aggregate equity holdings are constant over time, i.e., $A_{D t}^{s}=A_{D}^{s}$ and $A_{I t}^{s}=A_{I}^{s}$ for all $t$, and real asset prices are time-invariant linear functions of the aggregate dividend, i.e., $\phi_{t}^{s}=\phi^{s} y_{t}, \quad p_{t} \phi_{t}^{m} \equiv \bar{\phi}_{t}^{s}=\bar{\phi}^{s} y_{t}, \phi_{t}^{m} A_{I t}^{m}=Z y_{t}$, and $\phi_{t}^{m} A_{D t}^{m}=Z_{D} y_{t}$, where $Z, Z_{D} \in \mathbb{R}_{+}$Hence, in a recursive equilibrium, $\varepsilon_{t}^{*}=\bar{\phi}^{s}-\phi^{s} \equiv \varepsilon^{*}$, $\phi_{t+1}^{s} / \phi_{t}^{s}=\bar{\phi}_{t+1}^{s} / \bar{\phi}_{t}^{s}=\gamma_{t+1}, \phi_{t}^{m} / \phi_{t+1}^{m}=\mu / \gamma_{t+1}$, and $p_{t+1} / p_{t}=\mu$. Throughout the analysis, we let $\bar{\beta} \equiv \beta \bar{\gamma}$ and maintain the assumption $\mu>\bar{\beta}$ (but we consider the limiting case $\mu \rightarrow \bar{\beta}$ ).

For the analysis that follows, it is convenient to define

$$
\begin{aligned}
\hat{\mu} & \equiv \bar{\beta}\left[1+\frac{(1-\alpha \theta)(1-\bar{\beta} \delta)(\hat{\varepsilon}-\bar{\varepsilon})}{\hat{\varepsilon}}\right], \\
\bar{\mu} & \equiv \bar{\beta}\left[1+\frac{\alpha \theta(1-\bar{\beta} \delta)\left(\bar{\varepsilon}-\varepsilon_{L}\right)}{\bar{\beta} \delta \bar{\varepsilon}+(1-\bar{\beta} \delta) \varepsilon_{L}}\right]
\end{aligned}
$$

where $\hat{\varepsilon} \in\left[\bar{\varepsilon}, \varepsilon_{H}\right]$ is the unique solution to

$$
\bar{\varepsilon}-\hat{\varepsilon}+\alpha \theta \int_{\varepsilon_{L}}^{\hat{\varepsilon}}(\hat{\varepsilon}-\varepsilon) d G(\varepsilon)=0 .
$$

Lemma 4 (in online Appendix A) establishes that $\hat{\mu}<\bar{\mu}$. The following proposition characterizes the equilibrium set.

PROPOSITION 1: (i) A nonmonetary equilibrium exists for any parametrization. (ii) There is no recursive monetary equilibrium if $\mu \geq \bar{\mu}$. (iii) In the nonmonetary equilibrium, $A_{I}^{s}=A^{s}-A_{D}^{s}=A^{s}$ (only investors hold equity), there is no trade in the OTC market, and the equity price in the second subperiod is

$$
\phi_{t}^{s}=\phi^{s} y_{t}, \text { with } \phi^{s}=\frac{\bar{\beta} \delta}{1-\bar{\beta} \delta} \bar{\varepsilon} .
$$

(iv) If $\mu \in(\bar{\beta}, \bar{\mu})$, then there is one recursive monetary equilibrium; asset holdings of dealers and investors at the beginning of the OTC round of period $t$ are $A_{D t}^{m}=A_{t}^{m}-A_{I t}^{m}=0$ and

$$
A_{D}^{s}=A^{s}-A_{I}^{s} \begin{cases}=\delta A^{s} & \text { if } \bar{\beta}<\mu<\hat{\mu} \\ \in\left[0, \delta A^{s}\right] & \text { if } \mu=\hat{\mu} \\ =0 & \text { if } \hat{\mu}<\mu<\bar{\mu}\end{cases}
$$

and asset prices are

(8) $\phi_{t}^{s}=\phi^{s} y_{t}$, with $\phi^{s}= \begin{cases}\frac{\bar{\beta} \delta}{1-\bar{\beta} \delta} \varepsilon^{*} & \text { if } \bar{\beta}<\mu \leq \hat{\mu} \\ \frac{\bar{\beta} \delta}{1-\bar{\beta} \delta}\left[\bar{\varepsilon}+\alpha \theta \int_{\varepsilon_{L}}^{\varepsilon^{*}}\left(\varepsilon^{*}-\varepsilon\right) d G(\varepsilon)\right] & \text { if } \hat{\mu}<\mu<\bar{\mu},\end{cases}$ 


$$
\begin{aligned}
\bar{\phi}_{t}^{s} & =\bar{\phi}^{s} y_{t}, \text { with } \bar{\phi}^{s}=\varepsilon^{*}+\phi^{s}, \\
\phi_{t}^{m} & =Z \frac{y_{t}}{A_{t}^{m}}, \\
p_{t} & =\frac{\bar{\phi}^{s}}{Z} A_{t}^{m},
\end{aligned}
$$

where

$$
Z=\frac{\alpha G\left(\varepsilon^{*}\right) A_{I}^{s}+A_{D}^{s}}{\alpha\left[1-G\left(\varepsilon^{*}\right)\right]}\left(\varepsilon^{*}+\phi^{s}\right)
$$

and for any $\mu \in(\bar{\beta}, \bar{\mu}), \varepsilon^{*} \in\left(\varepsilon_{L}, \varepsilon_{H}\right)$ is the unique solution to

$$
\frac{(1-\bar{\beta} \delta) \int_{\varepsilon^{*}}^{\varepsilon_{H}}\left(\varepsilon-\varepsilon^{*}\right) d G(\varepsilon)}{\varepsilon^{*}+\bar{\beta} \delta\left[\bar{\varepsilon}-\varepsilon^{*}+\alpha \theta \int_{\varepsilon_{L}}^{\varepsilon^{*}}\left(\varepsilon^{*}-\varepsilon\right) d G(\varepsilon)\right] \mathbf{1}_{\{\hat{\mu}<\mu\}}}-\frac{\mu-\bar{\beta}}{\bar{\beta} \alpha \theta}=0 .
$$

(v) (a) As $\mu \rightarrow \bar{\mu}, \varepsilon^{*} \rightarrow \varepsilon_{L_{-}}$and $\phi_{t}^{s} \rightarrow(\bar{\beta} \delta /(1-\bar{\beta} \delta)) \bar{\varepsilon} y_{t} . \quad(b)$ As $\mu \rightarrow \bar{\beta}$, $\varepsilon^{*} \rightarrow \varepsilon_{H}$ and $\phi_{t}^{s} \rightarrow(\bar{\beta} \delta /(1-\bar{\beta} \delta)) \varepsilon_{H} y_{t}$.

In the nonmonetary equilibrium, dealers are inactive and equity shares are held only by investors. With no valued money, investors and dealers cannot exploit the gains from trade that arise from the heterogeneity in investor valuations in the first subperiod, and the real asset price is $\phi^{s}=(\bar{\beta} \delta /(1-\bar{\beta} \delta)) \bar{\varepsilon} y$, i.e., equal to the expected discounted value of the dividend stream since the equity share is not traded. (Shares can be traded in the Walrasian market of the second subperiod, but gains from trade at that stage are nil.) The recursive monetary equilibrium exists only if the inflation rate is not too high, i.e., if $\mu<\bar{\mu}$. In the monetary equilibrium, the marginal valuation, $\varepsilon^{*}$, which according to Lemma 1 partitions the set of investors into those who buy and those who sell the asset when they meet a dealer in the OTC market, is characterized by (13) in part (iv) of Proposition 1. Unlike what happens in the nonmonetary equilibrium, the OTC market is active in the monetary equilibrium, and it is easy to show that the marginal valuation, $\varepsilon^{*}$, is strictly decreasing in the rate of inflation, i.e., $\partial \varepsilon^{*} / \partial \mu<0$ (see Corollary 3 in the online Appendix). Intuitively, the real value of money falls as $\mu$ increases, so the marginal investor valuation, $\varepsilon^{*}$, decreases, reflecting the fact that under the higher inflation rate, the investor who was marginal under the lower inflation rate is no longer indifferent between carrying cash and equity out of the OTC market: he prefers equity.

According to Proposition $1,0 \leq \varepsilon_{L}<\varepsilon_{t}^{*}$ in the monetary equilibrium, so Lemma 1 implies that dealers hold no equity shares at the end of the OTC round: all equity is held by investors, in particular, by those investors who carried equity into the period but were unable to contact a dealer, and by those investors who purchased equity shares in bilateral trades with dealers. After the round of OTC trade, all the money supply is held by the investors who carried cash into the period but were unable to contact a dealer, by the investors who sold equity shares through dealers, and by those dealers who carried equity into the OTC market. 
A feature of the monetary equilibrium is that dealers never hold money overnight: at the beginning of every period $t$, the money supply is all in the hands of investors, i.e., $A_{D t}^{m}=0$ and $A_{I t}^{m}=A_{t}^{m}$. The reason is that access to the dealer market allows dealers to intermediate assets without cash. Whether it is investors or dealers who hold the equity shares overnight depends on the inflation rate: if it is low, i.e., if $\mu \in(\bar{\beta}, \hat{\mu})$, then only dealers hold equity overnight, that is, $\tilde{A}_{D t+1}^{s}=A^{s}$ and $\tilde{A}_{I t+1}^{s}=0$ for all $t$. Conversely, if the inflation rate is high, i.e., if $\mu \in(\hat{\mu}, \bar{\mu})$, then at the end of every period $t$, all equity shares are in the hands of investors, i.e., $\tilde{A}_{D t+1}^{s}=0$ and $\tilde{A}_{I t+1}^{s}=A^{s}$, so strictly speaking, in this case dealers only provide brokerage services in the OTC market. The intuition for this result is as follows. ${ }^{7}$ For dealers, the return from holding equity overnight is given by the resale price in the OTC market. If inflation is low, $\varepsilon_{t}^{*}$ is high (the asset is priced by relatively high valuation investors), and this means the resale price in the OTC market is high. Since dealers are sure to trade in the OTC market every period while investors only trade with effective probability $\alpha \theta$, the former are in a better position to reap the capital gains and end up holding all equity shares overnight. Conversely, if inflation is high then $\varepsilon_{t}^{*}$ is low, so the capital gain to a dealer from carrying the asset to sell in the OTC market is small. The benefit to investors from holding equity includes not only the resale value in the OTC market (which is small at high inflation) but also their own expected valuation of the dividend good, so for high inflation, the return that investors obtain from holding equity overnight is higher than it is for dealers. For example, as $\mu \rightarrow \bar{\mu}$ we have $\varepsilon_{t}^{*} \rightarrow \varepsilon_{L}$, so the dealer's expected return from holding equity overnight is $\left(\varepsilon_{L}+\phi^{s}\right) \bar{\gamma} / \phi^{s}$, while the investor's is $\left(\bar{\varepsilon}+\phi^{s}\right) \bar{\gamma} / \phi^{s}$.

Given the marginal valuation, $\varepsilon^{*}$, part (iv) of Proposition 1 gives all asset prices in closed form. The real ex dividend price of equity (in terms of the second subperiod consumption good), $\phi_{t}^{s}$, is given by (8). The cum-dividend dollar price of equity in the OTC market, $p_{t}$, is given by (11). The real price of money (in terms of the second subperiod consumption good), $\phi_{t}^{m}$, is given by (10). The real cum-dividend price of equity (in terms of the second subperiod consumption good) in the OTC market, $p_{t} \phi_{t}^{m}=\bar{\phi}^{s} y_{t}$, is given by (9).

Finally, part (va) states that as the rate of money creation increases toward $\bar{\mu}, \varepsilon^{*}$ approaches the lower bound of the distribution of valuations, $\varepsilon_{L}$, so no investor wishes to sell equity in the OTC market, and as a result the allocations and prices of the monetary equilibrium approach those of the nonmonetary equilibrium. Part (vb) states that as $\mu$ decreases toward $\bar{\beta}, \varepsilon^{*}$ increases toward the upper bound of the distribution of valuations, $\varepsilon_{H}$, so only investors with the highest valuation purchase equity in the OTC market (all other investors wish to sell it). Moreover, since $\bar{\beta}<\hat{\mu}$, as $\mu \rightarrow \bar{\beta}$ only dealers hold equity overnight. Thus, we have the following result.

COROLLARY 1: The allocation implemented by the recursive monetary equilibrium converges to the efficient allocation as $\mu \rightarrow \bar{\beta}$.

\footnotetext{
${ }^{7}$ See Lagos and Zhang (2015) for a more detailed discussion.
} 
Let $q_{t, k}^{B}$ denote the nominal price in the second subperiod of period $t$ of an $N$-period risk-free pure discount nominal bond that matures in period $t+k$, for $k=0,1,2, \ldots, N$ (so $k$ is the number of periods until the bond matures). Imagine the bond cannot be used as means of payment in the first subperiod. ${ }^{8}$ Then in a recursive monetary equilibrium, $q_{t, k}^{B}=(\bar{\beta} / \mu)^{k}$, and $i=\mu / \bar{\beta}-1$ is the time $t$ nominal yield to maturity of the bond with $k$ periods until maturity. Thus, the optimal monetary policy described in Corollary 1 and part ( $v b$ ) of Proposition 1 in which $\mu=\bar{\beta}$ can be interpreted as a policy that implements the Friedman rule, i.e., $i=0$ for all contingencies at all dates. Since the (gross) inflation rate is $\phi_{t}^{m} / \phi_{t+1}^{m}=\mu\left(y_{t} / y_{t+1}\right) \equiv 1+\pi_{t+1}, 1+i=\mu / \bar{\beta}$ is equivalent to

$$
1+i=(1+r)(1+\pi)
$$

with $1+\pi \equiv\left[E_{t} \frac{1}{1+\pi_{t+1}}\right]^{-1}=\mu / \bar{\gamma}$.

\section{Implications}

In this section, we discuss the main implications of the theory. Specifically, we show how asset prices and trade volume are determined by monetary policy and the details of the microstructure where the asset trades (e.g., the degree of market power of dealers and the ease with which investors find counterparties).?

\section{A. Asset Prices}

In this subsection, we study the asset-pricing implications of the theory. We focus on how the asset price depends on monetary policy and on the degree of OTC frictions as captured by the parameters that regulate trading frequency and the relative bargaining strengths of traders. ${ }^{10}$

The real price of equity in a monetary equilibrium is in part determined by the option available to low-valuation investors to resell the equity to high-valuation investors. If the growth rate of the money supply (and therefore the inflation rate) increases, equilibrium real money balances decline and the marginal investor valuation, $\varepsilon^{*}$, decreases, reflecting the fact that under the higher inflation rate, the investor valuation that was marginal under the lower inflation rate is no longer indifferent between carrying cash and equity out of the OTC market: he prefers equity. Since the marginal investor who prices equity in the OTC market has a lower valuation, the value of the resale option is smaller, i.e., the turnover liquidity of the asset is lower, which

\footnotetext{
${ }^{8}$ Notice that even though the bond cannot be traded for equity in the OTC round of trade, it can be exchanged (or redeemed) for money at the end of the period at no cost. Hence, how "illiquid" we deem this bond depends on the length of the model period. If, as in the quantitative analysis of Section V, the model period corresponds to one trading day, then the bond is in fact very liquid, or "very close to cash" according to the usual real-world standards.

${ }^{9}$ We focus on trade volume as a measure of financial liquidity because it will be the relevant variable in our empirical analysis. For completeness, in online Appendix Section A.A5 we show how conventional measures of financial liquidity other than trade volume (e.g., spreads, and dealer supply of immediacy) are determined by monetary policy and the details of the microstructure. In online Appendix Section A.A7 we also show that generically, asset prices in the monetary economy exhibit a speculative premium whose size varies systematically with monetary policy and the market microstructure.

${ }^{10}$ In Proposition 9 in the online Appendix we also establish the effect of a mean-preserving spread in the distribution of valuations on the equity price.
} 
in turn makes the real equity price (both the ex dividend price, $\phi^{s}$, and the cumdividend price, $\bar{\phi}^{s}$ ) smaller. Naturally, the real value of money, $\phi_{t}^{m}$, is also decreasing in the growth rate of the money supply. ${ }^{11}$ All of this is formalized in Proposition 2.

PROPOSITION 2: In the recursive monetary equilibrium: $(i) \partial \phi^{s} / \partial \mu<0$, (ii) $\partial \bar{\phi}^{s} / \partial \mu<0,\left(\right.$ iii) $\partial Z / \partial \mu<0$, and $\partial \phi_{t}^{m} / \partial \mu<0$.

Proposition 2 is useful for settings where monetary policy operates by changing the expected inflation rate, i.e., settings where changes in $i$ are associated exclusively with changes in $\pi$ in (14). The following result examines the behavior of real asset prices in settings where changes in $i$ are associated exclusively with changes in the real interest rate, $r .{ }^{12}$

PROPOSITION 3: In the recursive monetary equilibrium: $(i) \partial \phi^{s} / \partial r<0$, (ii) $\partial Z / \partial r<0$ and $\partial \phi_{t}^{m} / \partial r<0$.

In the OTC market, $\alpha$ is the probability an investor is able to contact a dealer, and $\theta$ is the investor's share of the gain from trade conditional on trading with a dealer. Hence, a larger $\alpha \theta$ implies a larger expected gain from trade for low-valuation investors when they sell the asset to dealers. In turn, this makes investors more willing to hold equity shares in the previous period, since they anticipate larger gains from selling the equity in case they were to draw a relatively low valuation in the following OTC round. Therefore, real equity prices, $\phi^{s}$ and $\bar{\phi}^{s}$, are increasing in $\alpha$ and $\theta .{ }^{13}$ If $\alpha$ increases, money becomes more valuable (both $Z$ and $\phi_{t}^{m}$ increase), provided we focus on a regime in which only investors carry equity overnight. ${ }^{14}$ Proposition 4 formalizes these ideas.

PROPOSITION 4: In the recursive monetary equilibrium: $(i) \partial \phi^{s} / \partial(\alpha \theta)>0$, (ii) $\partial \bar{\phi}^{s} / \partial(\alpha \theta)>0,($ iii $) \partial Z / \partial \alpha>0$ and $\partial \phi_{t}^{m} / \partial \alpha>0$, for $\mu \in(\hat{\mu}, \bar{\mu})$.

\section{B. Trade Volume}

Trade volume is commonly used as a measure of market liquidity because it is a manifestation of the ability of the market to reallocate assets across investors. According to Lemma 1 , any investor with $\varepsilon<\varepsilon_{t}^{*}$ who has a trading opportunity in the OTC market sells all his equity holding. Hence, in a recursive equilibrium, the quantity of assets sold by investors to dealers in the OTC market is

\footnotetext{
${ }^{11}$ The top row of online Appendix Figure A1 illustrates the typical time paths of the ex dividend equity price, $\phi_{t}^{s}$, real balances, $\phi_{t}^{m} A_{t}^{m}$, and the price level, $\phi_{t}^{m}$, for different values of $\mu$.

${ }^{12}$ The proof of Proposition 3, in online Appendix Section A.A4, is based on a continuous-time version of our discrete-time economy. The continuous-time formulation can be interpreted as an approximation to our baseline discrete-time model when the period length is small. Apart from allowing sharper analytical results, the continuous-time formulation is useful since the model period in our quantitative implementation of the theory is very short (specifically, a trading day; see Section VB for details).

${ }^{13}$ This finding is consistent with the behavior of the illiquidity premia in response to variations in the measures of liquidity documented by Ang, Shtauber, and Tetlock (2013).

${ }^{14}$ Real balances can actually fall with $\alpha$ for $\mu \in(\bar{\beta}, \hat{\mu})$. The bottom row of online Appendix Figure A1 illustrates the time paths of the ex dividend equity price, $\phi_{t}^{s}$, real balances $\phi_{t}^{m} A_{t}^{m}$, and the price level, $\phi_{t}^{m}$, for two different values of $\alpha$.
} 
$Q^{s}=\alpha G\left(\varepsilon^{*}\right) A_{I}^{s}$. From Lemma 1 , the quantity of assets purchased by investors from dealers is $Q^{b}=\alpha\left[1-G\left(\varepsilon^{*}\right)\right] A_{t}^{m} / p_{t}$. Thus, the total quantity of equity shares traded in the OTC market is $\mathcal{V}=Q^{b}+Q^{s}$, or equivalently ${ }^{15}$

$$
\mathcal{V}=2 \alpha G\left(\varepsilon^{*}\right) A_{I}^{s}+A_{D}^{s}
$$

Trade volume, $\mathcal{V}$, depends on the growth rate of the money supply, $\mu$, (or equivalently, expected inflation), the real interest rate, $r$, and dealers' market power $\theta$ indirectly, through the general equilibrium effect on $\varepsilon^{*}$. A decrease in $\mu$ or $r$, or an increase in $\theta$ increases the expected return to holding money relative to equity, which makes more investors willing to sell equity for money in the OTC market, i.e., $\varepsilon^{*}$ increases and so does trade volume, provided $G^{\prime}\left(\varepsilon^{*}\right)>0$. In other words, the increase in turnover liquidity caused by a decrease in $\mu$ or $r$, or an increase in $\theta$ will manifest itself through an increase in trade volume provided the cumulative distribution of investors is strictly increasing over the relevant range. The indirect positive effect on $\mathcal{V}$ (through $\varepsilon^{*}$ ) of an increase in the investors' trade probability $\alpha$ is similar to an increase in $\theta$, but in addition, $\alpha$ directly increases trade volume, since with a higher $\alpha$ more investors are able to trade in the OTC market. These results are summarized in the following proposition.

PROPOSITION 5: In the recursive monetary equilibrium, provided $G^{\prime}\left(\varepsilon^{*}\right)>0$ : (i) $\partial \mathcal{V} / \partial \mu<0,($ ii) $\partial \mathcal{V} / \partial r<0,($ iii) $\partial \mathcal{V} / \partial \theta>0$ and $\partial \mathcal{V} / \partial \alpha>0$.

\section{Discussion}

In this section we explain the economic rationale behind Propositions 2 and 3. To streamline the exposition, focus on a recursive monetary equilibrium in which investors carry equity overnight. In this case, the Euler equations for money and equity are

$$
\begin{aligned}
\phi_{t}^{m} & =\frac{1}{1+r} E_{t}\left[\phi_{t+1}^{m}+\alpha \theta \int_{\varepsilon^{*}}^{\varepsilon_{H}} \frac{\left(\varepsilon-\varepsilon^{*}\right) y_{t+1}}{p_{t+1}} d G(\varepsilon)\right], \\
\phi_{t}^{s} & =\frac{1}{1+r} \delta E_{t}\left[\bar{\varepsilon} y_{t+1}+\phi_{t+1}^{s}+\alpha \theta \int_{\varepsilon_{L}}^{\varepsilon^{*}}\left(\varepsilon^{*}-\varepsilon\right) y_{t+1} d G(\varepsilon)\right] .
\end{aligned}
$$

(See Corollary 2 in the online Appendix for details.) On the left side of (16), $\phi_{t}^{m}$ represents the cost (in terms of the second-subperiod consumption good) of purchasing an additional dollar in the competitive market of the second subperiod of period $t$. On the right side of (16) is the discounted expected value of the additional dollar in period $t+1$. This marginal value equals the expected value from holding the dollar for a whole period (until the competitive trading round at the end of period $t+1)$, i.e., $\phi_{t+1}^{m}$, plus the expected value of the option to exchange the dollar

\footnotetext{
${ }^{15}$ To obtain (15) we used the clearing condition for the dealer market, $Q^{b}=Q^{s}+A_{D t}^{s}$. Also, note that $\mathcal{V}$ is trade volume in the OTC market, but since every equity share traded in the first subperiod gets retraded in the second subperiod, total trade volume in the whole time period equals $2 \mathcal{V}$.
} 
for equity shares in the OTC round at the beginning of period $t+1$. This option is executed provided the investor gets access to the dealer market (with probability $\alpha$ ), gets a share $\theta$ of the gain from trade with the dealer, and gets a random valuation higher than $\varepsilon^{*}$, so the expected gain from exchanging the dollar for equity is $\alpha \theta \int_{\varepsilon^{*}}^{\varepsilon_{H}}\left(\varepsilon-\varepsilon^{*}\right) y_{t+1} / p_{t+1} d G(\varepsilon)$. Next, consider (17). On the left side, $\phi_{t}^{s}$ represents the cost (in terms of the second-subperiod consumption good) of purchasing an additional equity share in the competitive market of the second subperiod of period $t$. On the right side is the discounted (net of depreciation) expected value of the additional equity share in period $t+1$. This marginal value equals the expected cum-dividend value from holding the equity share for a whole period (until the competitive trading round at the end of period $t+1$ ), i.e., $\bar{\varepsilon} y_{t+1}+\phi_{t+1}^{s}$, plus the expected value of the option to resell the asset in the OTC round at the beginning of period $t+1$. This resale option is executed provided the investor gets access to the dealer market (with probability $\alpha$ ), gets a share $\theta$ of the gain from trade with the dealer, and gets a random valuation lower than $\varepsilon^{*}$, so the expected value of the resale option is $\alpha \theta \int_{\varepsilon_{L}}^{\varepsilon^{*}}\left(\varepsilon^{*}-\varepsilon\right) y_{t+1} d G(\varepsilon)$. This additional term that makes the equilibrium real value of an equity share larger than the discounted expected value of the dividend stream is reminiscent of the value of the resale option in Harrison and Kreps (1978). The novelty here is that the value of this resale option depends on monetary policy: through the effect that monetary policy has on asset reallocation and the determination of the marginal investor valuation, $\varepsilon^{*}$, in the OTC round.

Given $p_{t} \phi_{t}^{m} \equiv\left(\varepsilon^{*}+\phi^{s}\right) y_{t}, \phi_{t}^{m} / \phi_{t+1}^{m}=\mu / \gamma_{t+1}, E_{t} y_{t+1}=E_{t} \gamma_{t+1} y_{t}=\bar{\gamma} y_{t}$, and (14), the Euler equation (16) can be written as

$$
1=\frac{1}{1+i}\left[1+\alpha \theta \int_{\varepsilon^{*}}^{\varepsilon_{H}} \frac{\varepsilon-\varepsilon^{*}}{\varepsilon^{*}+\phi^{s}} d G(\varepsilon)\right] .
$$

The left side is the real cost of purchasing an additional unit of real money balances ( 1 unit of the numéraire good). The right side is the discounted expected value of bringing an additional unit of real money balances into the OTC round of the following period. Condition (18) makes clear that an increase in the nominal rate, $i$, acts as a tax on real money balances: it reduces the incentives to carry money, which in turn reduces the valuation of the marginal investor, $\varepsilon^{*}$. As a result, the real equity price falls, as is clear from (17). Notice that this result holds both, if the increase in the nominal rate is associated with an increase in expected inflation, $\pi$, as in part (i) of Proposition 2, and if the increase in the nominal rate is associated with an increase in the real interest rate, $r$, as in part (i) of Proposition 3. The intuitive reason why $\varepsilon^{*}$ falls in response to an increase in the nominal rate has to do with the fact that while in general equilibrium a change in $i$ affects investors' valuations of both equity and money, it affects the incentive to hold money relatively more.

To understand this, first suppose the increase in $i$ is associated with an increase in expected inflation, $\pi$, or equivalently, the money growth rate, $\mu$. The pricing equation (18) indicates that the first-order (partial equilibrium) effect of $\pi$ is to reduce the real value of a dollar, and that this is achieved by a reduction in $\varepsilon^{*}$, i.e., a reduction in the equilibrium cum-dividend value of an equity share that money is used to buy. The Euler equation for equity, (17) does not depend on $\pi$ directly; only indirectly through the general equilibrium effect of $\pi$ on real money balances. The direct effect of expected inflation on the real value of money is larger than the indirect effect on 
the price of equity, and the marginal investor valuation, $\varepsilon^{*}$, decreases as the investor that was marginal under the lower inflation rate tilts his portfolio away from money toward equity in an attempt to avoid the inflation tax.

For another angle on this logic, suppose the background monetary policy is the Friedman rule, as in Corollary 1. In this case the opportunity cost of holding money is 0 , i.e., $i=0$, so investors are willing to hold enough real money balances to satiate their random demand for liquidity in the OTC round, i.e., (18) implies $\varepsilon^{*}=\varepsilon_{H}$ if $i=0$. Intuitively, this means that the highest valuation investors (those with valuations equal to $\varepsilon_{H}$ ) who contact dealers are able to absorb the whole supply of equity shares in the dealer market, and the equilibrium equity price reflects only their valuation. If the inflation rate is higher than the target level prescribed by the Friedman rule, then the opportunity cost of holding money is positive, and therefore the highest valuation investors are budget constrained in the OTC round as they no longer choose to hold enough real money balances to be financially unconstrained for every realization of the next-period valuation (formally, (18) implies $\varepsilon^{*}<\varepsilon_{H}$ if $i>0)$. Since investors with valuation $\varepsilon_{H}$ are no longer able to absorb the whole asset supply traded in the dealer market, investors with lower valuations are now able to purchase some equity shares, and therefore the marginal investor who prices equity has valuation $\varepsilon^{*}<\varepsilon_{H}$. In sum, as $\pi$ increases, aggregate real balances fall, investors with relatively high valuations become less able to express their valuations, and as a result, the asset is held, and priced, by investors with lower valuations.

To conclude, suppose the increase in $i$ is associated with an increase in the real rate, $r$. In this case we have shown that $\varepsilon^{*}$ also falls, again reflecting the fact that an increase in the real rate reduces the value of money relatively more than the value of an equity share. The reason is that, since equity dominates money in terms of rate of return (equity yields a real dividend and money does not), an equilibrium with valued money necessarily requires that money be a relatively better store of value than equity (formally, $\mu<\bar{\mu}$ ). This means that while an increase in $r$ reduces investors' valuations of both equity and money, it has a relatively smaller effect on the incentive to hold equity, which necessarily has a more "front-loaded" payoff structure than money in the monetary equilibrium.

\section{Empirical Analysis}

According to the theory, the real asset price decreases in response to an entirely unanticipated and permanent increase in the nominal interest rate (part (i) of Proposition 2 and part (i) of Proposition 3, together with (14)). The mechanism through which the increase in the nominal rate is transmitted to the asset price is a reduction in turnover liquidity, i.e., a reduction in the resale option value, accompanied by a nonpositive change in trade volume (parts (i) and (ii) of Proposition 5). These two theoretical results suggest two hypotheses that can be tested with price and turnover data: (i) surprise increases in the nominal rate reduce the marketwide stock return (and possibly trade volume), and (ii) the strength of the mechanism depends on the turnover liquidity of the stocks (e.g., as proxied for by the turnover rate of the stock). The following proposition (based on a generalization of the theory with multiple assets indexed by $s \in\{1,2, \ldots, N\}$ that differ in terms of the trading frequency, $\alpha^{s}$ ) provides a formal theoretical basis for these two hypotheses. 
PROPOSITION 6: In the recursive monetary equilibrium, $(i) \partial \log \phi^{s} / \partial \pi<0$, (ii) $\partial \log \phi^{s} / \partial r<0,($ iii $) \partial^{2} \log \phi^{s} /\left(\partial \alpha^{s} \partial \pi\right)<0,\left(\right.$ iv) $\partial^{2} \log \phi^{s} /\left(\partial \alpha^{s} \partial r\right)<0$.

Thus, theory predicts a negative semi-elasticity of the real equity price with respect to the nominal interest rate, regardless of whether the increase in the nominal rate is associated with an increase in the expected inflation rate, $\pi$, or an increase in the real rate, $r$ (parts (i) and (ii) of Proposition 6). Moreover, since the component of the asset price associated with the expected value of the resale option discussed in Section IIIC is increasing in the turnover of the asset, and turnover is an increasing function of $\alpha^{s}$, theory predicts that the magnitude of this semi-elasticity is larger for stocks with higher turnover (parts (iii) and (iv) of Proposition 6).

\section{A. Data}

We use daily time series for all individual common stocks in the New York Stock Exchange (NYSE) from the Center for Research in Security Prices (CRSP). ${ }^{16}$ The daily stock return from CRSP takes into account changes in prices and accrued dividend payment, i.e., the return of stock $s$ on day $t$ is $\mathcal{R}_{t}^{s}=\left(\left(\left(P_{t}^{s}+D_{t}^{s}\right) / P_{t-1}^{s}\right)-1\right) \times 100$, where $P_{t}^{s}$ is the ex dividend dollar price of stock $s$ on day $t$, and $D_{t}^{s}$ denotes the dollar dividend paid per share of stock $s$ on day $t$. As a measure of trade volume for each stock, we construct the daily turnover rate from CRSP, i.e., $\mathcal{T}_{t}^{s}=\mathcal{V}_{t}^{s} / A_{t}^{s}$, where $\mathcal{V}_{t}^{s}$ is the trade volume of stock $s$ on day $t$ (measured as the total number of shares traded) and $A_{t}^{s}$ is the number of outstanding shares of stock $s$ on day $t$. Whenever we use an average, e.g., of equity returns or turnover rates across a set of stocks, we use the arithmetic average, e.g., $\mathcal{R}_{t}^{I}=(1 / n) \sum_{s=1}^{n} \mathcal{R}_{t}^{s}$ and $\mathcal{T}_{t}^{I}=(1 / n) \sum_{s=1}^{n} \mathcal{T}_{t}^{s}$ are the average return and the average turnover rate for the universe of $n$ common stocks listed in the NYSE. ${ }^{17}$

As a proxy for the policy (nominal interest) rate, we use the rate on the nearest Eurodollar futures contract due to mature after the FOMC policy announcement, as in Rigobon and Sack (2004)..$^{18}$ Specifically, we use the 3-month Eurodollar futures rate produced by the Chicago Mercantile Exchange Group (CME Group) and supplied by Datastream. In some of our empirical estimations, we use the tick-by-tick nominal interest rate implied by 30-day federal funds futures and

\footnotetext{
${ }^{16}$ We report results for NASDAQ stocks in online Appendix Section D.D3.

${ }^{17}$ We report results for value-weighted returns in online Appendix Section D.D4.

${ }^{18}$ Eurodollar futures are based on a $\$ 1$ million face value 3-month maturity Eurodollar time deposit. These futures contracts mature during the conventional IMM (International Monetary Market) dates in the months of March, June, September, or December, extending outward 10 years into the future. In addition, at any point in time, there are so-called 3-month Eurodollar serial contracts extending 4 months into the future that mature in months that are not conventional IMM dates. For example, at the beginning of January 2016, there are contracts maturing in mid-March, mid-June, mid-September, and mid-December 2016, through 2025. There are also serial contracts maturing in mid-January, mid-February, mid-April, and mid-May 2016. Thus, depending on the timing of the FOMC announcement, the nearest contract to mature may expire between 0 and 30 days after the announcement. Current quotes are available at http://www.cmegroup.com/trading/interest-rates/stir/eurodollar_quotes_ settlements_futures.html. An advantage of using a futures rate as a proxy for the "policy rate" is that its movement on dates of FOMC policy announcements reflects policy surprises only and does not reflect anticipated policy changes. The importance of focusing on the surprise component of policy announcements (rather than on the anticipated component) in order to identify the response of asset prices to monetary policy was originally pointed out by Kuttner (2001) and has been emphasized by the literature since then, e.g., Bernanke and Kuttner (2005) and Rigobon and Sack (2004). Gürkaynak, Sack, and Swanson (2007) offers empirical evidence supporting the use of futures contracts as an effective proxy for policy expectations and discusses their use to define policy shocks.
} 
consider a high-frequency measure of the unexpected change in the nominal policy rate in a narrow 30-minute time window around the FOMC announcement. The sample we analyze runs from January 3, 1994 to December 31, 2007. ${ }^{19}$ The sample includes between 1,300 and 1,800 stocks (depending on the time period) and 133 FOMC announcement dates. ${ }^{20}$

In the following subsections, we use the data described above to estimate the sign and magnitude of the effect of monetary policy on stock returns and turnover. In Subsection IVB, we estimate these effects for FOMC announcement days for a broad index of stocks. In Subsection IVC, we document that the strength of the effect of monetary policy on stock returns differs systematically with the turnover liquidity of the stock. In Subsection IVD, we go a step further and estimate the dynamic effects of the policy announcement on returns and turnover.

\section{B. Aggregate Announcement-Day Effects}

The empirical literature has followed several approaches to estimate the impact of monetary policy on the stock market. A popular one, known as event-study analysis, consists of estimating the market reaction to monetary policy surprises on a subsample of trading days consisting exclusively of the days of FOMC announcements (we denote this subsample $S_{1}$ ). Let $i_{t}$ denote the day $t$ "policy rate" (in our case, the CME Group 3-month Eurodollar future with closest expiration date at or after day $t$, expressed in percentage terms) and define $\Delta i_{t} \equiv i_{t}-i_{t-1}$. The event-study analysis consists of running the following regression:

$$
Y_{t}^{I}=a+b \Delta i_{t}+\epsilon_{t}
$$

for $t \in S_{1}$, with $Y_{t}^{I}=\mathcal{R}_{t}^{I}$, where $\epsilon_{t}$ is an exogenous shock to the asset price. ${ }^{21} \mathrm{We}$ refer to the estimator $b$ as the event-study estimator (or E-based estimator, for short).

A concern with (19) is that it does not take into account the fact that the policy rate on the right side may itself be reacting to asset prices (a simultaneity bias) and that a number of other variables (e.g., news about economic outlook) are likely to have an impact on both the policy rate and asset prices (an omitted variables bias). This concern motivates us to also consider two other estimators: the heteroskedasticity-based

\footnotetext{
${ }^{19}$ We start our sample period in 1994 because prior to 1994, policy changes in the federal funds target were unannounced and frequently occurred between FOMC meetings. From 1994 onward, all changes are announced and most coincided with FOMC meetings, so as policy announcement dates we use the dates of FOMC meetings obtained from the web site of the Board of Governors of the Federal Reserve System (http://www.federalreserve. gov/monetarypolicy/fomccalendars.htm). See Bernanke and Kuttner (2005) for more discussion on the exact timing of policy announcements.

${ }^{20}$ Our full sample contains 135 policy dates. We discard two dates: 9/13/2001 and 9/17/2001 (the two atypical FOMC announcements in the immediate aftermath of $9 / 11 / 2001)$. One of our estimation procedures requires data involving first differences in variables on the policy day and on the day preceding the policy day. In that case, we follow Rigobon and Sack (2004) and discard three additional policy dates because they are preceded by either one or two holidays in financial markets. Another of our estimation procedures relies on high-frequency market activity in a narrow time interval around the exact time of the monetary policy announcement. In this case, we use the data from Gorodnichenko and Weber (2016) that consist of 118 scheduled policy dates. For each trading day, we discard observations whose return or turnover rate on that given day is in the top or bottom one percentile.

${ }^{21}$ In the context of monetary policy, this approach was originally used by Cook and Hahn (1989) and has been followed by a large number of papers, e.g., Bernanke and Kuttner (2005), Cochrane and Piazzesi (2002), Kuttner (2001), and Thorbecke (1997).
} 
estimator (H-based estimator, for short) proposed by Rigobon and Sack (2004), and a version of the event-study estimator that relies on an instrumental variable identification strategy that uses intraday high-frequency tick-by-tick interest rate data. The H-based estimator identifies the response of asset prices based on the heteroskedasticity of monetary policy shocks. The high-frequency instrumental variable estimator (HFIV estimator, for short) addresses the omitted variable bias and the concern that the Eurodollar futures rate may itself respond to market conditions on policy announcement days, by focusing on changes in a proxy for the policy rate in a very narrow 30-minute window around the time of the FOMC announcement. ${ }^{22}$ Table 1 presents the baseline results. The first column corresponds to the event-based estimation, the second column corresponds to the heteroskedasticity-based estimation, and the third column corresponds to the high-frequency instrumental variable estimation. Returns are expressed in percentage terms. The first row presents estimates of the reaction of the marketwide NYSE return to monetary policy. The point estimate for $b$ in (19) is -5.47 . This means that a 1 basis point (bp) increase in the policy rate causes a decrease of 5.47 basis points (bps) in the stock market return on the day of the policy announcement. ${ }^{23}$ The analogous H-based point estimate is -11.31 . These results are in line with those reported in previous studies. ${ }^{24}$ The HFIV point estimate is -9.38 , implying that a 25 bp surprise increase in the policy rate causes a decrease in the stock market return of 2.34 percentage points (pps) on the day of the policy announcement. ${ }^{25}$ Figure 1 shows a scatterplot with the unexpected change in the policy rate (measure by the high-frequency change in the fed funds future rate) on the horizontal axis, and the announcement-day marketwide stock return on the vertical axis, both expressed in bps. The negative relationship between stock returns and fed funds rate surprises is readily visible from the fitted line.

Previous studies have not clearly identified the specific economic mechanism that transmits monetary policy shocks to the stock market. Conventional asset-pricing theory suggests three broad immediate reasons why an unexpected policy nominal rate increase may lead to a decline in stock prices. It may be associated with a decrease in expected dividend growth, with a rise in the future real interest rates used to discount dividends, or with an increase in the expected excess returns (i.e., equity premia) associated with holding stocks. Our theory formalizes a new mechanism: the reduction in turnover liquidity caused by the increase in the opportunity cost of holding the nominal assets that are routinely used to settle financial transactions. To begin assessing this mechanism, we again estimate $b$ in (19), and the analogous

\footnotetext{
${ }^{22}$ In the online Appendix we discuss the derivation of the H-based estimator (Section B.B1) and describe the construction of the HFIV estimator (Section B.B2).

${ }^{23}$ The $R^{2}$ indicates that 14 percent of the variance of equity prices in days of FOMC policy announcements is associated with news about monetary policy.

${ }^{24}$ The comparable event-based estimates in Bernanke and Kuttner (2005), which focuses on a different sample period and measures stock returns using the value-weighted return from CRSP, range between -2.55 and -4.68 . The comparable heteroskedasticity-based estimates in Rigobon and Sack (2004), which uses a different series for the Eurodollar forward rate, are -6.81 for the S\&P 500 index, -6.5 for the WIL5000 index, -9.42 for the NASDAQ, and -4.85 for the DJIA.

${ }^{25}$ In comparing the E-based, H-based, and HFIV estimates, one should bear in mind that the number of policy dates varies slightly between the three estimation methods, as explained in footnote 20 .
} 
Table 1-Empirical Response of NYSE Marketwide Stock Returns and Turnover to Monetary Policy

\begin{tabular}{|c|c|c|c|c|c|c|}
\hline & \multicolumn{2}{|c|}{ E-based } & \multicolumn{2}{|c|}{ H-based } & \multicolumn{2}{|c|}{ HFIV } \\
\hline & Estimate & SD & Estimate & SD & Estimate & SD \\
\hline Return & -5.47 & 1.30 & -11.31 & 4.53 & -9.38 & 2.86 \\
\hline Turnover & -0.0021 & 0.0013 & -0.0015 & 0.0034 & -0.0052 & 0.0013 \\
\hline
\end{tabular}

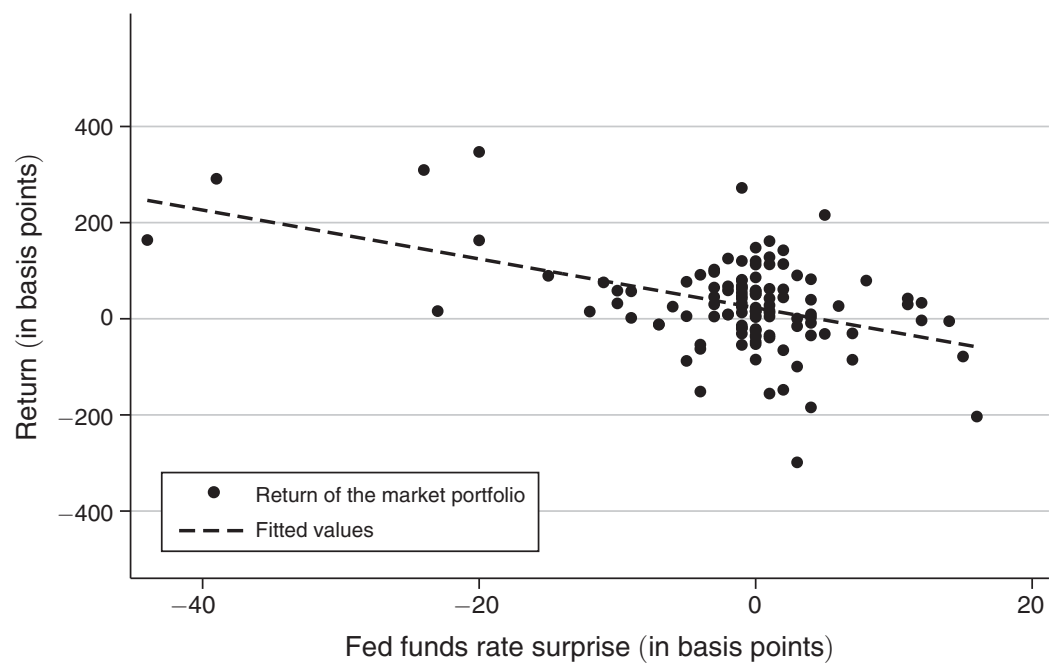

Figure 1. Unexpected Component of the Change in the Policy Rate on FOMC Announcement Dates and ANNOUnCEMENT-Day Marketwide NYSE Stock RETURnS

H-based and HFIV estimates, but with $Y_{t}^{I}=\mathcal{T}_{t}^{I}-\mathcal{T}_{t-1}^{I}$, i.e., we use the change in the daily turnover rate averaged over all traded stocks as the dependent variable.

The estimated effects of monetary policy announcements on the daily marketwide NYSE turnover rate are reported in the second row of Table 1. According to the E-based estimate, a $100 \mathrm{bp}$ increase in the policy rate causes a change in the level of the marketwide turnover rate on the day of the policy announcement equal to $-0.0021 .^{26}$ The daily marketwide turnover rate for our sample period is 0.0048 (i.e., on average, stocks turn over 1.22 times during a typical year composed of 252 trading days), which means that according to the E-based estimate, an increase in the policy rate of 25 bps causes a reduction in the marketwide turnover rate on the day of the policy announcement of about 10 percent of its typical level. The HFIV estimate for a $100 \mathrm{bp}$ increase in the policy rate is -0.0052 , implying that a $25 \mathrm{bp}$ increase in the policy rate causes a reduction in the marketwide turnover rate of about 27 percent of its typical level.

\footnotetext{
${ }^{26}$ The $R^{2}$ indicates that 3 percent of the variance of the daily turnover rate in days of FOMC policy announcements is associated with unexpected changes in monetary policy.
} 


\section{Disaggregative Announcement-Day Effects}

Another way to inspect the turnover-liquidity transmission mechanism of monetary policy is to exploit the cross-sectional variation in turnover rates that exists across stocks. Our theory implies that the magnitude of the change in the stock return induced by a change in the policy rate will depend on the turnover liquidity of the stock (e.g., as measured by the turnover rate of the stock). To test this prediction, we sort stocks into portfolios according to their turnover liquidity, as follows. For each FOMC announcement date, $t$, we calculate $\mathcal{T}_{t}^{s}$ as the average turnover rate of an individual stock $s$ over all trading days during the four weeks prior to the day of the policy announcement. We then sort all stocks into 20 portfolios by assigning stocks with $\mathcal{T}_{t}^{s}$ ranked between the $[5(i-1)]$ th percentile and $(5 i)$ th percentile to the $i$ th portfolio, for $i=1, \ldots, 20$. Hence, the average turnover rate over the four-week period prior to the announcement date for a stock in the $i$ th portfolio is at least as large as that of a stock in the $(i-1)$ th portfolio. In Table 2, the Turnover column reports the annual turnover rate (based on 252 trading days per year) corresponding to each of the 20 portfolios. For example, portfolio 1 turns over 0.17 times per year while portfolio 20 turns over 3.57 times per year. ${ }^{27}$

For each of the 20 portfolios, Table 2 reports the E-based, H-based, and HFIV estimates of the announcement-day responses of the return to a 1 percentage point (pp) increase in the policy rate. All the estimates are negative, as predicted by the theory. Also, the magnitude of the (statistically significant) estimates increases with the turnover liquidity of the portfolio. For example, according to the HFIV estimates, a $1 \mathrm{bp}$ increase in the policy rate causes a decrease of $6.44 \mathrm{bps}$ in the return of portfolio 1 and a decrease of $16.40 \mathrm{bps}$ in the return of portfolio 20. For all three estimation methods, the relative differences in responses across portfolios are of similar magnitude. For example, the response of the return of the most liquid portfolio is about 2.5 times larger than the response of the least liquid portfolio. ${ }^{28}$ Figure 2 shows the announcement-day returns of portfolio 1 (the crosses) and portfolio 20 (the circles), along with their respective fitted lines. The larger magnitude of the response of the more liquid portfolio is evident.

As an alternative way to estimate the heterogeneous responses of returns to monetary policy shocks for stocks with different turnover liquidity, we ran an event-study regression of individual stock returns (for the universe of stocks listed in the NYSE) on changes in the policy rate, an interaction term between the change in the policy rate and individual stock daily turnover rate, and several controls. As before, $\Delta i_{t}$ denotes the monetary policy shock on policy announcement day $t$ (measured by

\footnotetext{
${ }^{27}$ Our motivation for constructing these liquidity-based portfolios is twofold. First, at a daily frequency, individual stock returns are extremely noisy; by grouping stocks into portfolios based on some characteristic(s) related to returns, it becomes possible to see average return differences. Second, stock-specific turnover measures are time-varying, i.e., the turnover rate of a particular stock may change over time. Bernanke and Kuttner (2005) also examines the responses of more disaggregated indices to monetary policy shocks. Specifically, they estimate the responses of 10 industry portfolios constructed from CRSP returns as in Fama and French (1988) but find that the precision of their estimates is not sufficient to reject the hypothesis of an equal reaction for all 10 industries.

${ }^{28}$ In online Appendix Section B.B3, we report similar results from an alternative procedure that sorts stocks into portfolios according to the strength of individual stock returns to changes in an aggregate (marketwide) measure of turnover. This alternative sorting criterion allows us to control for other differences across stocks, such as the conventional risk factors used in empirical asset-pricing models.
} 
Table 2-Empirical Responses of Stock Returns to Monetary Policy for NYSE LiQuidity PorTFolios (1994-2007 SAMPLE)

\begin{tabular}{|c|c|c|c|c|c|c|c|}
\hline \multirow[b]{2}{*}{ Portfolio } & \multirow[b]{2}{*}{ Turnover } & \multicolumn{2}{|c|}{ E-based } & \multicolumn{2}{|c|}{ H-based } & \multicolumn{2}{|c|}{ HFIV } \\
\hline & & Estimate & SD & Estimate & SD & Estimate & SD \\
\hline 1 & 0.17 & -3.74 & 0.98 & -7.18 & 2.76 & -6.44 & 1.80 \\
\hline 2 & 0.32 & -3.81 & 1.03 & -8.40 & 3.66 & -5.81 & 2.03 \\
\hline 3 & 0.43 & -3.94 & 1.47 & -7.49 & 3.99 & -6.40 & 2.81 \\
\hline 4 & 0.52 & -3.63 & 1.51 & -7.58 & 4.02 & -6.61 & 2.99 \\
\hline 5 & 0.59 & -3.97 & 1.23 & -9.98 & 4.78 & -5.80 & 2.55 \\
\hline 6 & 0.66 & -4.06 & 1.10 & -9.36 & 4.26 & -6.01 & 2.29 \\
\hline 7 & 0.73 & -4.96 & 1.10 & -11.41 & 4.76 & -7.37 & 2.38 \\
\hline 8 & 0.80 & -4.56 & 1.23 & -9.83 & 4.41 & -7.74 & 2.93 \\
\hline 9 & 0.87 & -4.25 & 1.58 & -9.53 & 4.80 & -7.56 & 3.48 \\
\hline 10 & 0.94 & -5.32 & 1.19 & -11.27 & 4.58 & -8.74 & 2.82 \\
\hline 11 & 1.01 & -5.63 & 1.30 & -11.05 & 4.47 & -9.32 & 2.85 \\
\hline 12 & 1.11 & -5.53 & 1.39 & -10.39 & 4.24 & -9.67 & 3.11 \\
\hline 13 & 1.21 & -5.93 & 1.31 & -11.68 & 4.33 & -10.09 & 2.45 \\
\hline 14 & 1.32 & -5.81 & 1.30 & -12.24 & 4.56 & -9.95 & 2.75 \\
\hline 15 & 1.45 & -6.84 & 1.58 & -12.72 & 4.42 & -11.46 & 2.89 \\
\hline 16 & 1.60 & -6.60 & 1.62 & -13.38 & 5.02 & -12.31 & 3.52 \\
\hline 17 & 1.79 & -7.22 & 1.57 & -15.44 & 5.79 & -12.66 & 3.41 \\
\hline 18 & 2.07 & -7.41 & 1.76 & -14.80 & 5.53 & -13.31 & 3.78 \\
\hline 19 & 2.50 & -7.52 & 2.01 & -14.70 & 5.75 & -14.10 & 4.35 \\
\hline 20 & 3.57 & -8.62 & 2.15 & -17.85 & 6.66 & -16.40 & 4.56 \\
\hline NYSE & 1.22 & -5.47 & 1.30 & -11.31 & 4.53 & -9.38 & 2.86 \\
\hline
\end{tabular}

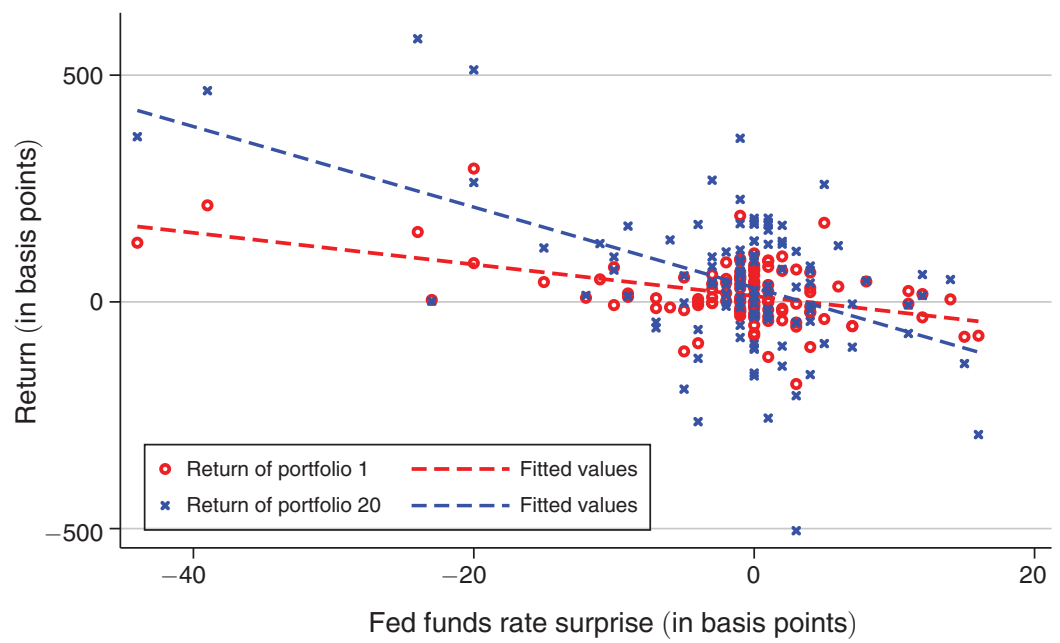

Figure 2. Unexpected Component of the Change in the Policy Rate on FOMC Announcement Dates and Announcement-Day NYSE Stock Returns for Portfolio 1 (Circles) and Portfolio 20 (Crosses)

the change between day $t$ and day $t-1$ in the 3-month Eurodollar futures contract with nearest expiration after the day $t$ FOMC policy announcement), and $\mathcal{T}_{t}^{s}$ is the average turnover rate of the individual stock $s$ over all the trading days during the four weeks prior to the day of the policy announcement of day $t$. Let $\Delta i$ and $\mathcal{T}$ denote 
the sample averages of $\Delta i_{t}$ and $\mathcal{T}_{t}^{s}$, respectively, and define $\overline{\mathcal{T}_{t}^{s}} \equiv\left(\mathcal{T}_{t}^{s}-\mathcal{T}\right)$ and $\overline{\Delta i_{t}} \equiv\left(\Delta i_{t}-\Delta i\right)$. The regression we fit is

$$
\begin{aligned}
\mathcal{R}_{t}^{s}= & \beta_{0}+\beta_{1} \Delta i_{t}+\beta_{2} \mathcal{T}_{t}^{s}+\beta_{3} \overline{\mathcal{T}_{t}^{s}} \times \overline{\Delta i_{t}} \\
& +D_{s}+D_{t}+\beta_{4}\left(\Delta i_{t}\right)^{2}+\beta_{5}\left(\mathcal{T}_{t}^{s}\right)^{2}+\varepsilon_{s t},
\end{aligned}
$$

where $D_{s}$ is a stock fixed effect, $D_{t}$ is a quarterly time dummy, and $\varepsilon_{s t}$ is the error term corresponding to stock $s$ on policy announcement day $t$. The time dummies control for omitted variables that may affect the return of all stocks in the NYSE over time. The stock fixed effects control for the effects that permanent stock characteristics not included explicitly in the regression may have on individual stock returns. We include the interaction term $\overline{\mathcal{T}_{t}^{s}} \times \overline{\Delta i_{t}}$ to estimate how the effect of changes in the policy rate on individual stock returns varies across stocks with different turnover liquidity. The coefficient of interest is $\beta_{3}$, i.e., we want to test whether changes in the policy rate affect individual stock returns through the stock-specific turnover-liquidity channel. The estimate of $\beta_{3}$ can help us evaluate whether increases (reductions) in the policy rate cause larger reductions (increases) in returns of stocks with a larger turnover rate, i.e., whether $\beta_{3}<0$.

Table 3 reports the results from estimating nine different specifications based on (20). Specification (I) excludes $D_{s}, D_{t}$, the interaction term, $\overline{\mathcal{T}_{t}^{s}} \times \overline{\Delta i_{t}}$, and the squared terms, $\left(\Delta i_{t}\right)^{2}$ and $\left(\mathcal{T}_{t}^{s}\right)^{2}$. Specification (II) adds the interaction term to specification (I). Specification (III) adds $D_{s}$ to specification (II). Specification (IV) adds $D_{t}$ to specification (II). Specification (V) adds $D_{s}$ to specification (IV). Specifications (VI), (VII), (VIII), and (IX) each add the squared terms $\left(\Delta i_{t}\right)^{2}$ and $\left(\mathcal{T}_{t}^{s}\right)^{2}$ to specifications (II), (III), (IV), and (V), respectively. In all specifications, all estimates are significant at 1 percent level.

The estimates of $\beta_{1}$ lie near -5.5 in all specifications, implying that a $1 \mathrm{bp}$ increase in the policy rate reduces the return of a stock with average turnover by about 5.5 bps on the day of the policy announcement. ${ }^{29}$ The estimate of interest, $\beta_{3}$, is large and negative in all specifications. The negative and statistically significant estimates of $\beta_{3}$ indicate that the magnitude of the negative effect of unexpected changes in the policy rate on announcement-day equity returns is larger for stocks with higher turnover liquidity. To interpret the magnitude of the estimates, consider a stock $A$ with a daily turnover rate equal to 0.014 (i.e., a stock in liquidity portfolio 20) and an equity $B$ with an annual turnover rate equal to 0.0007 (i.e., a stock in liquidity portfolio 1). Then, for example, according to specification (IX), the estimate of $\beta_{3}$ is -487 , implying that a $1 \mathrm{bp}$ increase in the policy rate reduces the announcement-day return by $\beta_{1}+2 \beta_{4}+\beta_{3}\left(\mathcal{T}_{t}^{A}-\mathcal{T}\right) \approx-10$ bps for equity $A$ and by $\beta_{1}+2 \beta_{4}+\beta_{3}\left(\mathcal{T}_{t}^{B}-\mathcal{T}\right) \approx-3$ bps for equity $B$. These estimates are quite close to the E-based estimates for portfolio 20 and for portfolio 1 reported in Table 2. Together with the findings reported in Table 1 and Table 2, the results in Table 3

\footnotetext{
${ }^{29}$ Recall the average daily turnover in our sample is 0.0048 .
} 
Table 3-Effects of Monetary Policy on Stock Returns of Individual NYSE Stocks

\begin{tabular}{|c|c|c|c|c|c|c|c|c|c|}
\hline Variable & (I) & (II) & (III) & (IV) & $(\mathrm{V})$ & (VI) & (VII) & (VIII) & (IX) \\
\hline$\Delta i_{t}$ & $\begin{array}{c}-5.37 \\
(0.11)\end{array}$ & $\begin{array}{c}-5.56 \\
(0.11)\end{array}$ & $\begin{array}{c}-5.60 \\
(0.13)\end{array}$ & $\begin{array}{c}-5.83 \\
(0.12)\end{array}$ & $\begin{array}{c}-5.82 \\
(0.13)\end{array}$ & $\begin{array}{l}-5.21 \\
(0.099)\end{array}$ & $\begin{array}{c}-5.23 \\
(0.098)\end{array}$ & $\begin{array}{l}-5.30 \\
(0.110)\end{array}$ & $\begin{array}{l}-5.30 \\
(0.112)\end{array}$ \\
\hline $\mathcal{T}_{t}^{s}$ & $\begin{array}{l}2,894 \\
(148)\end{array}$ & $\begin{array}{l}3,094 \\
(147)\end{array}$ & $\begin{array}{l}2,920 \\
(181)\end{array}$ & $\begin{array}{l}1,256 \\
(160)\end{array}$ & $\begin{array}{c}589 \\
(207)\end{array}$ & $\begin{array}{l}4,389 \\
(315)\end{array}$ & $\begin{array}{l}4,454 \\
(370)\end{array}$ & $\begin{array}{l}2,832 \\
(333)\end{array}$ & $\begin{array}{l}2,190 \\
(407)\end{array}$ \\
\hline$\overline{\mathcal{T}^{s}} \times{\overline{\Delta i_{t}}}$ & & $\begin{array}{l}-553 \\
(29)\end{array}$ & $\begin{array}{c}-557 \\
(30)\end{array}$ & $\begin{array}{c}-492 \\
(30)\end{array}$ & $\begin{array}{l}-491 \\
(32)\end{array}$ & $\begin{array}{l}-554 \\
(29)\end{array}$ & $\begin{array}{l}-560 \\
(31)\end{array}$ & $\begin{array}{l}-485 \\
(30)\end{array}$ & $\begin{array}{l}-487 \\
(32)\end{array}$ \\
\hline$D_{s}$ & & & yes & & yes & & yes & & yes \\
\hline$D_{t}$ & & & & yes & yes & & & yes & yes \\
\hline$\left(\Delta i_{t}\right)^{2}$ & & & & & & $\begin{array}{l}0.04 \\
(0.008)\end{array}$ & $\begin{array}{l}0.04 \\
(0.008)\end{array}$ & $\begin{array}{l}0.05 \\
(0.009)\end{array}$ & $\begin{array}{l}0.05 \\
(0.010)\end{array}$ \\
\hline$\left(\mathcal{T}_{t}^{s}\right)^{2}$ & & & & & & $\begin{array}{c}-53,852 \\
(14,751)\end{array}$ & $\begin{array}{l}-57,208 \\
(15,093)\end{array}$ & $\begin{array}{l}-63,775 \\
(14,887)\end{array}$ & $\begin{array}{l}-56,275 \\
(15,597)\end{array}$ \\
\hline$R^{2}$ & 0.0241 & 0.0286 & 0.0286 & 0.0778 & 0.0778 & 0.0290 & 0.0290 & 0.0783 & 0.0784 \\
\hline
\end{tabular}

Notes: Both the interest rate and stock returns are expressed in basis points. Each column reports the coefficients from a separate pooled OLS regression based on (20). Number of observations: 205,760. Standard errors reported in parentheses. All estimates are significant at the 1 percent level.

provide additional evidence that turnover liquidity is a quantitatively important channel that transmits monetary policy shocks to asset prices. ${ }^{30}$

\section{Dynamic Effects}

In the previous section we documented the effect of monetary policy shocks on equity returns and turnover on the day the policy announcement takes place. While the turnover liquidity channel highlighted by our theory can generate the effects on announcement days documented in the previous section, the theoretical channel is eminently dynamic. In the theory, persistent changes in the nominal rate affect stock returns because they imply persistent changes in the future resale value of the stock. To study the dynamic effects of monetary policy on prices and turnover rates, we conduct a vector autoregression (VAR) analysis on the sample consisting of all trading days between January 3, 1994 and December 31, 2007.

The baseline VAR we estimate consists of three variables, i.e., $\left\{i_{t}, \mathcal{R}_{t}^{I}, \mathcal{T}_{t}^{I}\right\}$, where $i_{t}, \mathcal{R}_{t}^{I}$, and $\mathcal{T}_{t}^{I}$ are the daily measures of the policy rate, the stock return, and turnover described in Sections IVA and IVB. ${ }^{31}$ The lag length is set to $10 .{ }^{32}$ To

\footnotetext{
${ }^{30}$ In online Appendix Section D.D2 we show that our estimates of $\beta_{3}$ based on (20) are robust to including a number of additional controls, such as sensitivity to the three most common Fama-French factors, industry dummies, and a firm-specific measure of leverage (reliance on bank debt). We also ran regression (20) (on the whole sample, 1994-2007) including stock and time fixed effects and dropping all other controls, and found an estimate of $\beta_{3}$ equal to -339.61 (significant at the 1 percent level). The estimates of $\beta_{3}$ in Table 3 are significant at the 5 percent level if the standard error is clustered by calendar date.

${ }^{31}$ In Section IVB, we used the change in the 3-month Eurodollar futures rate on the day of the FOMC announcement as a proxy for the unexpected component of the change in the true policy rate, i.e., the effective federal funds rate. In this section, we instead regard the 3-month Eurodollar futures rate as the policy rate itself. We do this because, at a daily frequency, the effective federal funds rate is very volatile for much of our sample, e.g., due to institutional considerations, such as "settlement Wednesdays." The path of the 3-month Eurodollar futures rate is quite similar to the effective federal funds rate, but it does not display the large regulation-induced weekly swings. In any case, we have also performed the estimation in this section using the daily effective federal funds rate instead of the Eurodollar futures rate, and the results for returns and turnover are quite similar.

${ }^{32}$ The Akaike information criterion (AIC) suggests 10 lags, while Schwarz's Bayesian information criterion (SBIC) and the Hannan and Quinn information criterion (HQIC) suggest 5 lags. We adopted the formulation with 10 lags, but both formulations deliver similar estimates.
} 
Policy rate

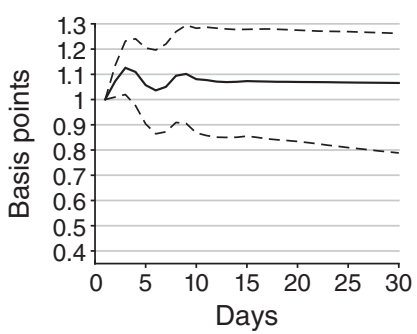

Policy rate

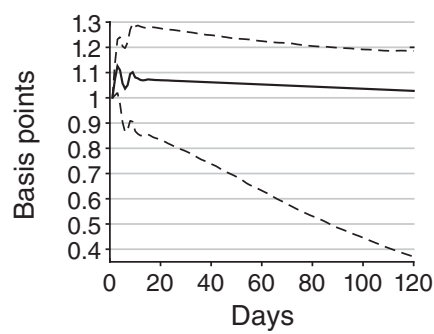

Cumulative return

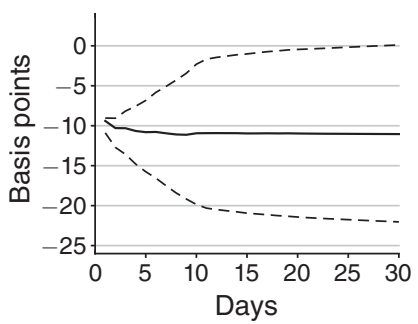

Cumulative return

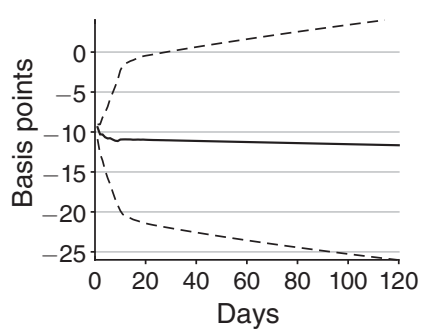

Daily turnover rate

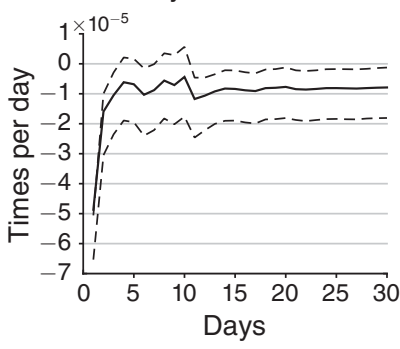

Daily turnover rate

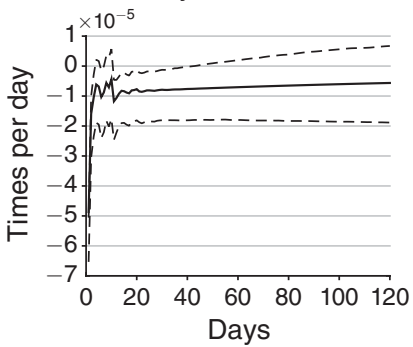

Figure 3. Empirical Impulse Responses to a 1 Basis Point Increase in the Policy Rate

Notes: Solid lines are point estimates. Broken lines are the 95 percent confidence bands.

identify the effects of monetary policy shocks, we apply an identification scheme based on an external high-frequency instrument. ${ }^{33}$

Figure 3 reports the impulse responses of the policy rate, the average cumulative stock return between day $t$ and day $t+j$ defined by $\overline{\mathcal{R}}_{t, t+j}^{I} \equiv \prod_{s=1}^{j} \mathcal{R}_{t+s}^{I}$, and the average turnover rate, to a $1 \mathrm{bp}$ increase in the policy rate. ${ }^{34}$ The 99 percent confidence intervals for $\left\{i_{t}, \mathcal{R}_{t}^{I}, \mathcal{T}_{t}^{I}\right\}$ are computed using a recursive wild bootstrap based on 10,000 replications. ${ }^{35}$ The top and bottom rows show responses for forecast horizons of 30 days and 120 days, respectively. The path of the policy rate is very persistent (it remains significantly above the level prevailing prior to the shock for about 18 months). The middle panels in Figure 3 show the response of daily cumulative stock returns. On impact, in response to the $1 \mathrm{bp}$ unexpected increase in the nominal rate, the stock return falls by about 9.4 bps. The magnitude of this response on the day of the policy shock is basically the same as the HFIV point estimate reported in Table 1. The negative effect on the stock price is persistent (the upper bound of the 99 percent confidence remains below zero for about 30 days). The right panels

\footnotetext{
${ }^{33}$ See online Appendix Section B.B4 for details. The basic idea of structural vector autoregression (SVAR) identification using instruments external to the VAR can be traced back to Romer and Romer (1989) and has been adopted in a number of more recent papers, including Cochrane and Piazzesi (2002), Hamilton (2003), Kilian (2008a, b), Stock and Watson (2012), Mertens and Ravn (2013), and Gertler and Karadi (2015).

${ }^{34}$ The impulse response for the cumulative return illustrates the path of $\left\{\overline{\mathcal{R}}_{-1, j-1}^{I}\right\}$, where $j=1,2, \ldots$ indexes the number of days after the policy announcement.

${ }^{35}$ The procedure is described in online Appendix Section B.B4. See Gonçalves and Kilian (2004) for a formal econometric analysis of this method. We compute the confidence bands for $\left\{\overline{\mathcal{R}}_{t, t+j}^{I}\right\}$ by compounding the confidence bands of the return response $\left\{\mathcal{R}_{t}^{I}\right\}$ (i.e., in the same way we compute $\left\{\overline{\mathcal{R}}_{t, t+j}^{I}\right\}$ from $\left\{\mathcal{R}_{t}^{I}\right\}$ ).
} 
in Figure 3 show the response of the level of the daily turnover rate. On impact, a 1 bp surprise increase in the nominal rate causes a change in the level of the turnover rate equal to -0.00005 , which is the same as the HFIV point estimate reported in Table 1. According to the estimated impulse response, it takes about 1 day for the turnover rate to recover one-half of the initial drop. However, beyond that point, the negative effect of the increase in the policy rate on turnover is persistent (e.g., it takes 43 days for it to become statistically insignificant).

In order to inspect the turnover-liquidity transmission mechanism further, we exploit the cross-sectional variation in turnover rates across stocks and carry out the same VAR analysis of this section but individually on each of 20 liquidity portfolios of stocks, sorted on turnover liquidity. ${ }^{36}$ Figure 4 shows the estimated impulse responses (to a $1 \mathrm{bp}$ unexpected increase in the policy rate) of the cumulative returns of each of the 20 liquidity portfolios for a forecast horizon of 30 days. In the figure, the darker impulse responses correspond to the portfolios with higher turnover liquidity (e.g., the lightest impulse response is for portfolio 1 and the darkest, for portfolio 20). To further illustrate the results, Figure 5 reports the impulse responses and the corresponding 99 percent confidence intervals of the cumulative portfolio return to a $1 \mathrm{bp}$ unexpected increase in the policy rate for a forecast horizon of 30 days, for portfolios 1, 10, and 20. Notice that the announcement-day portfolio-by-portfolio responses estimated by the VAR line up well with the portfolio-by-portfolio HFIV estimates reported in Table 2. As in Section IVC, we again find that on the announcement day, the negative responses of returns to an unexpected increase in the nominal rate tend to be larger in magnitude for portfolios with higher turnover liquidity. However, here these responses appear to be estimated much more precisely than in Table 2. ${ }^{37}$ Also, notice that, as will be the case in the quantitative theory, the price responses of the portfolios with larger turnover liquidity are not only larger in magnitude on impact, but also tend to be more persistent. ${ }^{38}$

In this section we have provided empirical evidence consistent with the turnover-liquidity transmission mechanism of monetary policy: a persistent increase in the nominal rate reduces the resale value of stocks, and this reduction in turnover liquidity is reflected in a persistent price reduction and higher future stock returns. ${ }^{39}$

\footnotetext{
${ }^{36}$ In Section IVC we re-sorted stocks into liquidity portfolios for each day in our sample of FOMC announcement dates (based on the average daily turnover rate over the four weeks prior to each FOMC announcement). For the high-frequency VAR that we estimate in this section, stocks are resorted into one of 20 liquidity portfolios every day. On days with no FOMC announcement, the sorting is based on daily turnover rate. On FOMC announcement days, stocks are sorted based on their turnover rate two days prior to the announcement. Since the ranking of a given stock in terms of turnover tends to be quite persistent, all the sorting schemes described here deliver similar results.

${ }^{37}$ Aside from the fact that the VAR specification is more flexible than (19), our VAR estimation also relies on the HFIV identification scheme. In fact, notice that even for the simple specification (19), Table 1 and Table 2 show that in general, the HFIV identification strategy by itself already delivers estimates that are more precise and more statistically significant than the E-based and H-based estimates.

${ }^{38}$ Based on the announcement-day evidence alone, one might conjecture that the differential return response on impact across liquidity portfolios may simply reflect that the prices of stocks with lower turnover liquidity take longer to react to the FOMC shock. This conjecture, however, does not seem to be supported by the VAR evidence in Figures 4 and 5. The conjecture is also not supported by the additional regression analysis we carry out in online Appendix Section D.D1, where we estimate the effect of an unexpected policy shock on day $t$ on $\overline{\mathcal{R}}_{t-1, t+1}^{I}$, i.e., the cumulative stock return for the two-day horizon after the policy announcement.

${ }^{39}$ Bernanke and Kuttner (2005) is one of a few papers that has tried to identify the economic forces behind the negative effect of nominal rate increases on stock returns. They use a VAR to decompose excess equity returns into components attributable to news about dividends, real interest rates, and future excess returns. They find the component associated with future excess returns accounts for the largest part of the response, i.e., an increase in the policy rate lowers stock prices mostly by increasing the expected equity premium. Bernanke and Kuttner speculate
} 


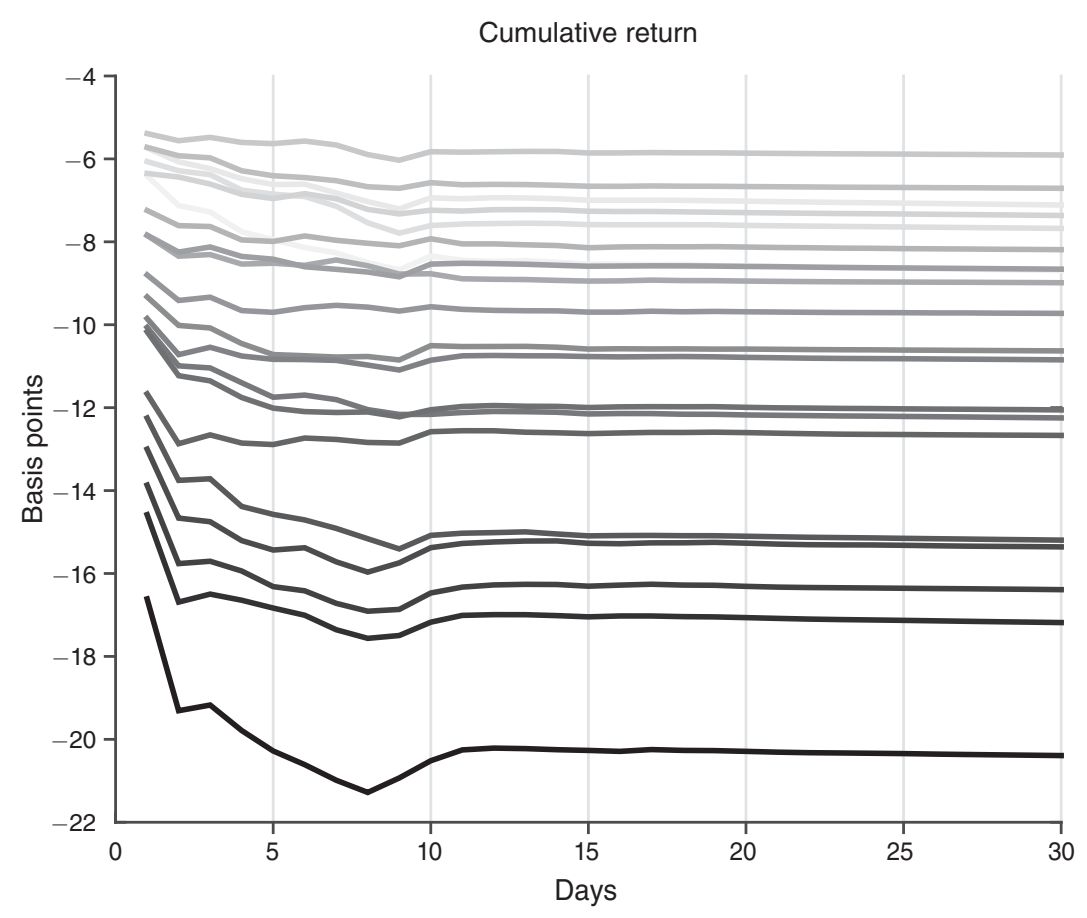

Figure 4. Empirical Impulse Responses of Cumulative Stock Returns FOR THE 20 NYSE LIQUIDITY PORTFOLIOS

Notes: The figure reports responses to a 1 basis point increase in the policy rate. Darker shades correspond to more liquid portfolios.

\section{Quantitative Analysis}

The theoretical results we used to motivate the empirical analysis of Section IV (e.g., part (i) of Proposition 2, part (i) of Proposition 3, and parts (i) and (ii) of Proposition 5) correspond to a permanent, unanticipated increase in the nominal rate, which, while suggestive, is somewhat different from the policy shocks underlying the empirical estimates of Section IV. Thus in order to assess the predictions and quantitative performance of the theory, in this section we formulate, calibrate, and simulate a generalized version of the model of Section I.

We generalize the model along three dimensions. First, we incorporate aggregate uncertainty in the path of monetary policy, represented by changes in the nominal interest rate implemented via open-market operations. This extension allows us to consider theoretical experiments that resemble more closely what goes on in financial markets, in the sense that while investors may be surprised by the timing and size of changes in the nominal rate, they take into account a probability distribution over future paths of the monetary policy so these changes are not 
Cumulative return

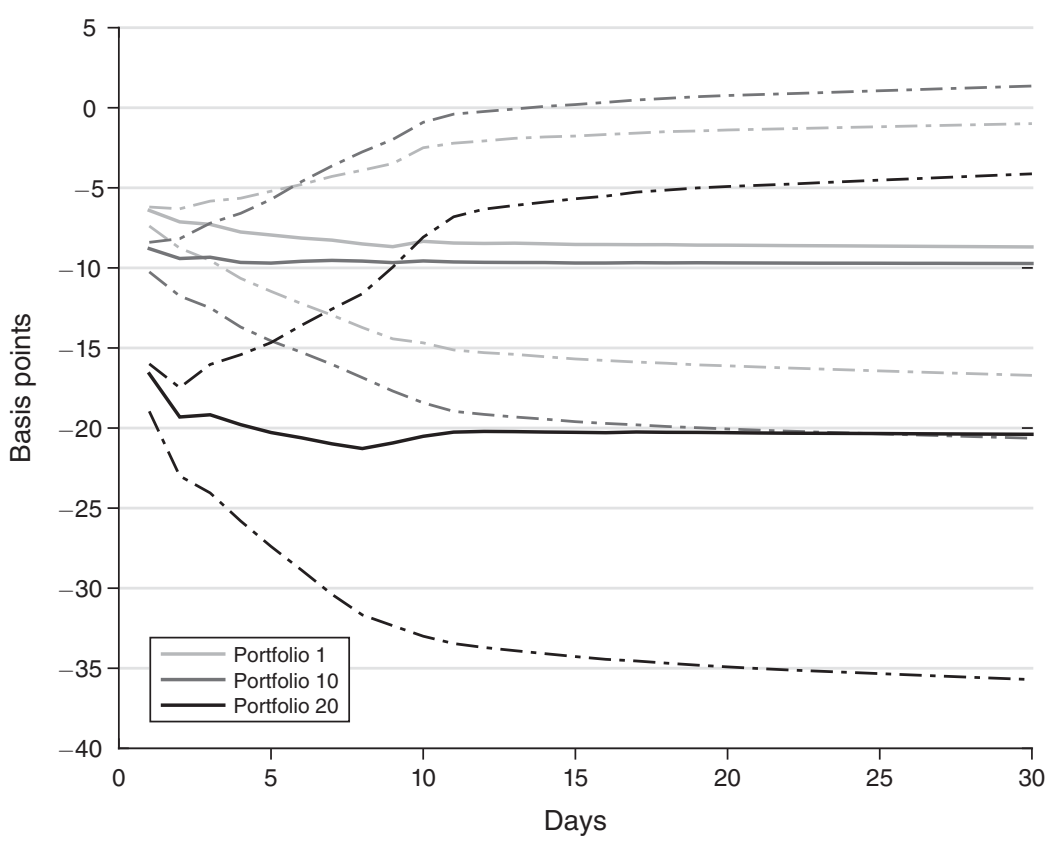

Figure 5. Empirical Impulse Responses of Cumulative Stock Returns FOR SELECTEd NYSE LiQuidity PoRTFolios

Notes: The figure reports responses to a 1 basis point increase in the policy rate. Solid lines are point estimates. Broken lines are the 95 percent confidence bands.

entirely unexpected. Second, we extend the model so that the innovations to the nominal policy rate may be associated with innovations to the expected inflation rate (as in the propositions of Section III), as well as with innovations to the real interest rate. This extension allows us to quantify the turnover-liquidity mechanism for settings where changes in the nominal rate may be associated with changes in the expected inflation rate as well as with changes in the real rate. Third, we extend the model to the case of multiple equity classes that differ in their liquidity properties. This extension allows us to provide additional evidence for the turnover-liquidity mechanism by exploiting the cross-sectional heterogeneity and using it to assess the quantitative theoretical effects of monetary policy on the cross section of equity returns.

\section{A. Generalized Model}

There are $N$ equity classes, each indexed by $s \in \mathbb{N}=\{1,2, \ldots, N\}$. The outstanding quantity of equity shares of class $s$ is $A^{s}$. Since the focus is on the implication of liquidity differences across equity classes, we assume each class gives the same dividend $y_{t}$, which follows the same stochastic process described in the one-asset model of Section I. An investor's period $t$ valuation of the dividend of any equity is distributed independently over time and across investors, with cumulative distribution function $G$, just as in the one-asset setup. 
We model liquidity differences as follows. In each round of OTC trade, each investor can trade equity class $s \in \mathbb{N}$ with probability $\alpha^{s} \in[0,1]$. The event that the investor is able to trade equity class $s$ is independent of the event that he is able to trade any other equity class $n \in \mathbb{N}$. We interpret $\alpha^{s}$ as the probability that an individual investor contacts a dealer with whom he can trade equity class $s$. This captures the idea that dealers are specialized in trading a particular equity class. ${ }^{40}$ In the OTC trading round there is a competitive dealer market for each equity class. These markets are segmented in the following sense: (i) in the OTC trading round, equity $s$ can only be traded in market $s$, and (ii) at the beginning of the period, investors partition the money they will use for trading stocks in the first subperiod into a cash portfolio with $N$ components, i.e., $\left\{a_{t}^{m s}\right\}_{s \in \mathbb{N}}$, where $a_{t}^{m s}$ is the amount of money the investor chooses to have available to trade equity class $s$ in the OTC market of period $t$. Each investor makes this cash rebalancing decision after having observed the realization of the aggregate state, but before learning which equity classes he will be able to trade, and before learning his individual valuation of the dividend (the last two assumptions keep the ex post number of investor types to a minimum). For simplicity, in this section we assume dealers do not hold asset inventories overnight (and without loss, also that they do not hold money overnight).

In Section I, we assumed a constant growth rate of the money supply, i.e., $A_{t+1}^{m}=\mu A_{t}^{m}$, where $\mu \in \mathbb{R}_{++}$. In this section we broaden the analysis of monetary policy along three dimensions. (i) We allow the monetary authority to inject or withdraw money not only with lump sum taxes, but also via open-market operations. This is a more realistic implementation of monetary policy, and makes the theory more flexible in that it can encompass a wider range of responses to monetary policy shocks. (ii) We model monetary policy as a stochastic process. This allows the theory to exhibit monetary policy shocks that resemble the policy surprises in the empirical analysis of Section IV. (iii) We allow monetary policy to affect market outcomes by influencing the nominal rate through both of its components: the expected inflation rate, and the real interest rate. This allows us to assess the robustness of the turnover-liquidity mechanism to different degrees of pass-through from nominal rates to real rates. In summary, we will consider general monetary policy processes that consist of three components: an open-market operation, a change in expected inflation, and a change in the real rate. Each of these components of the monetary policy process is modeled as follows.

In the first subperiod, each investor can always trade in a competitive market where the monetary authority sells $B_{t}$ one-period risk-free pure-discount nominal bonds. A bond issued in the first subperiod of $t$ yields one dollar with certainty in the following subperiod. The dollar price of a bond in this market is denoted $q_{t}$. The bond market is segmented in the same way as the markets for equity shares,

\footnotetext{
${ }^{40}$ In the theory, differences in $\alpha, \theta$, or $G$ all give rise to differences in turnover across assets. We focus on differences in $\alpha$ because it is conceptually the simplest and analytically the most direct way to construct asset classes that differ in turnover liquidity. However, one could carry out the theoretical analysis by constructing asset classes based on differences in $G$ and $\theta$. Differences in $G$ work similarly to differences in $\alpha$ (see the equivalence result proved in Proposition 10 in the online Appendix). With regard to differences in $\theta$, in a large class of models that includes this one, Duffie, Gârleanu, and Pedersen (2005) and Lagos and Rocheteau (2009), the equilibrium asset price does not depend on $\alpha$ and $\theta$ independently, but on their product, $\alpha \theta$. Thus, for asset-pricing purposes, differences in $\alpha$ can be interpreted as capturing differences in the trading probability or in the bargaining power. The quantitative response of turnover to money shocks will typically depend on whether assets differ in $\alpha$ or $\theta$, however.
} 
i.e., at the beginning of period $t$, having observed the realization of the monetary policy variables (but before knowing which equity classes he will be able to trade or his dividend valuation), each investor chooses a partition of his money holdings, $\left\{a_{t}^{m s}\right\}_{s \in \overline{\mathbb{N}}}$, where $\overline{\mathbb{N}} \equiv \mathbb{N} \cup\{b\}$, and $a_{t}^{m b}$ denotes the amount of money the investor chooses to have available to trade bonds in the first subperiod of $t$. The size of the bond issue, $B_{t}$, relative to the size of the beginning-of-period money supply, $A_{t}^{m}$, is denoted $\omega_{t}$. That is, if there are $A_{t}^{m}$ dollars outstanding at the beginning of period $t$, in the bond market of the first subperiod $t$ the government sells claims to $B_{t}=\omega_{t} A_{t}^{m}$ dollars payable in the following subperiod.

The beginning-of-period money supply evolves according to

$$
A_{t+1}^{m}=\left[1+\left(1-q_{t}\right) \omega_{t}\right] \tilde{\mu}_{t} A_{t}^{m}
$$

where $\tilde{\mu}_{t} \in \mathbb{R}_{++}$denotes the growth rate of the money supply between the end of period $t$ and the beginning of period $t+1$ (implemented via lump-sum transfers in the second subperiod of $t$ ). The monetary authority can implement any arbitrary process for the growth rate of the beginning-of-period money supply, i.e., can set, $A_{t+1}^{m}=\mu_{t} A_{t}^{m}$ for any positive path $\left\{\mu_{t}\right\}_{t=1}^{\infty}$, despite the random changes in the money supply induced by the open-market operations. ${ }^{41}$ Finally, to allow for the possibility that monetary policy can affect outcomes by influencing the real rate as well as expected inflation, we generalize the constant interest rate $r$ of Section I to a stochastic process $\left\{r_{t}\right\}_{t=1}^{\infty}$.

To summarize, we model monetary policy as a stochastic process $\left\{\boldsymbol{\tau}_{t}\right\}_{t=1}^{\infty}$, where $\tau_{t} \equiv\left(\omega_{t}, \mu_{t}, r_{t}\right)$. This formulation is general enough to encompass situations where monetary policy amounts to changing expected inflation (as in monetarist models) as well as settings where monetary policy amounts to directly influencing real rates (as in New Keynesian models). We assume $\left\{\boldsymbol{\tau}_{t}\right\}_{t=1}^{\infty}$ follows a Markov chain with transition matrix $\sigma_{i j}=\operatorname{Pr}\left(\tau_{t+1}=\tau_{j} \mid \tau_{t}=\tau_{i}\right)$, where $\boldsymbol{\tau}_{i} \equiv\left(\omega_{i}, \mu_{i}, r_{i}\right) \in \mathbb{R}_{++}^{3}$ and $\tau_{j} \equiv\left(\omega_{j}, \mu_{j}, r_{j}\right) \in \mathbb{R}_{++}^{3}$ for $i, j \in \mathcal{M}=\{1, \ldots, M\}$. The realization of $\tau_{t}$ is known at the beginning of period $t$.

We specialize the analysis to recursive equilibria in which prices and portfolio decisions are time-invariant functions of an aggregate state vector that follows a time-invariant law of motion. The state vector is $\mathbf{x}_{t}=\left(A_{t}^{m}, y_{t}, \boldsymbol{\tau}_{t}\right) \in \mathbb{R}_{+}^{5}$. Asset prices in a recursive equilibrium will be denoted $\phi_{t}^{s}=\phi^{s}\left(\mathbf{x}_{t}\right), \bar{\phi}_{t}^{s}=\bar{\phi}^{s}\left(\mathbf{x}_{t}\right)$, $\phi_{t}^{m}=\phi^{m}\left(\mathbf{x}_{t}\right), p_{t}^{s}=p^{s}\left(\mathbf{x}_{t}\right), q_{t}=q\left(\mathbf{x}_{t}\right)$, and $\varepsilon_{t}^{s *}=\varepsilon^{s *}\left(\mathbf{x}_{t}\right)$. Let $A_{t}^{m k}$ denote the amount of money that investors have available to trade asset $k \in \overline{\mathbb{N}}$ at the beginning of period $t$ (i.e., the bond, if $k=b$, or equity, if $k \in \mathbb{N}$ ). The laws of motion for the state variables $A_{t}^{m}, y_{t}$, and $\tau_{t}$ are exogenous (as described above), while $A_{t}^{m k}=\Psi^{k}\left(\mathbf{x}_{t}\right)$ for $k \in \overline{\mathbb{N}}$, where the decision rule $\Psi^{k}$ is determined in equilibrium. Suppose $\mathbf{x}_{t}=\left(A_{t}^{m}, y_{t}, \boldsymbol{\tau}_{i}\right)$ and focus on a recursive equilibrium with the property that real prices are linear functions of the aggregate dividend, and $\Psi^{k}\left(\mathbf{x}_{t}\right)=\lambda_{i}^{k} A_{t}^{m}$ for all $k \in \overline{\mathbb{N}}$, where $\lambda_{i}^{s} \in[0,1]$ denotes the fraction of the

\footnotetext{
${ }^{41}$ Specifically, $\tilde{\mu}_{t}=\mu_{t} /\left[1+\left(1-q_{t}\right) \omega_{t}\right]$ implies $A_{t+1}^{m}=\mu_{t} A_{t}^{m}$ for any $\left\{\mu_{t}\right\}_{t=1}^{\infty}$. The government budget constraint is $B_{t}+T_{t} / \phi_{t}^{m}=A_{t+1}^{m}-\left(A_{t}^{m}-q_{t} B_{t}\right)$, so the real lump-sum transfer (expressed in terms of the second-subperiod consumption good) needed to implement $A_{t+1}^{m}=\mu_{t} A_{t}^{m}$ is $T_{t}=\left[\left(\mu_{t}-1\right)-\left(1-q_{t}\right) \omega_{t}\right] \phi_{t}^{m} A_{t}^{m}$.
} 
beginning-of-period money holdings that investors have chosen to have available to trade asset class $k$ in the OTC round of period $t$. Then, $\phi^{s}\left(\mathbf{x}_{t}\right)=\phi_{i}^{s} y_{t}$, $\bar{\phi}^{s}\left(\mathbf{x}_{t}\right) \equiv p^{s}\left(\mathbf{x}_{t}\right) \phi^{m}\left(\mathbf{x}_{t}\right)=\bar{\phi}_{i}^{s} y_{t}, \phi^{m}\left(\mathbf{x}_{t}\right) A_{t}^{m}=Z_{i} y_{t}, \quad q\left(\mathbf{x}_{t}\right)=q_{i}$, and $\varepsilon^{s *}\left(\mathbf{x}_{t}\right) \equiv$ $\left[\bar{\phi}^{s}\left(\mathbf{x}_{t}\right)-\phi^{s}\left(\mathbf{x}_{t}\right)\right] / y_{t}=\bar{\phi}_{i}^{s}-\phi_{i}^{s} \equiv \varepsilon_{i}^{s *}$.

In online Appendix Section C.C3, we show that an equilibrium is characterized by a vector $\left\{\phi_{i}^{s}, \varepsilon_{i}^{s *}, Z_{i}, \lambda_{i}^{s}\right\}_{i \in \mathcal{M}, s \in \overline{\mathbb{N}}}$ of $M(3 N+2)$ unknowns that solves the following system with $M(3 N+2)$ independent equations:

$$
\begin{aligned}
\phi_{i}^{s} & =\frac{\bar{\gamma} \delta}{1+r_{i}} \sum_{j \in \mathcal{M}} \sigma_{i j}\left[\bar{\varepsilon}+\phi_{j}^{s}+\alpha^{s} \theta \int_{\varepsilon_{L}}^{\varepsilon_{j}^{s *}}\left(\varepsilon_{j}^{s *}-\varepsilon\right) d G(\varepsilon)\right], \\
Z_{i} & =\frac{\bar{\gamma}}{\left(1+r_{i}\right) \mu_{i}} \sum_{j \in \mathcal{M}} \sigma_{i j}\left[1+\alpha^{s} \theta \int_{\varepsilon_{j}^{s *}}^{\varepsilon_{H}} \frac{\varepsilon-\varepsilon_{j}^{s *}}{\varepsilon_{j}^{s *}+\phi_{j}^{s}} d G(\varepsilon)\right] Z_{j},
\end{aligned}
$$

$$
\max \left(\omega_{i} / \lambda_{i}^{b}, 1\right)=1+\alpha^{s} \theta \int_{\varepsilon_{i}^{s *}}^{\varepsilon_{H}} \frac{\varepsilon-\varepsilon_{i}^{s *}}{\varepsilon_{i}^{s *}+\phi_{i}^{s}} d G(\varepsilon) \quad \text { for all }(i, s) \in \mathcal{M} \times \mathbb{N}
$$

$$
Z_{i} \lambda_{i}^{s}=\frac{G\left(\varepsilon_{i}^{s *}\right) A^{s}}{1-G\left(\varepsilon_{i}^{s *}\right)}\left(\varepsilon_{i}^{s *}+\phi_{i}^{s}\right) \quad \text { for all }(i, s) \in \mathcal{M} \times \mathbb{N}
$$

$$
1-\lambda_{i}^{b}=\sum_{s \in \mathbb{N}} \lambda_{i}^{s} \text { for all } i \in \mathcal{M}
$$

In the following subsections, we calibrate and simulate this model to assess the ability of the theory to account for the empirical findings reported in Section IV. Before doing so, it is useful to define the theoretical analogues of the variables we studied in the empirical section.

The return of stock $s$ at date $t+1$ is $\mathcal{R}_{t+1}^{s}=\bar{\phi}_{t+1}^{s} / \phi_{t}^{s}-1$, where $\bar{\phi}_{t}^{s} \equiv p_{t} \phi_{t}^{m}=\phi_{t}^{s}+\varepsilon_{t}^{*} y_{t}$ is the cum-dividend price of equity at date $t$ defined in Section II. The real return from holding a dollar between the end of period $t$ and the end of period $t+1$ is $\phi_{t+1}^{m} / \phi_{t}^{m} \equiv\left(1+\pi_{t+1}\right)^{-1}$, where $\pi_{t+1}$ denotes the (net) inflation rate between $t$ and $t+1$. In a recursive equilibrium, suppose the state is $\mathbf{x}_{t}=\left(A_{t}^{m}, y_{t}, \boldsymbol{\tau}_{j}\right)$ at $t$, and $\mathbf{x}_{t+1}=\left(\mu_{j} A_{t}^{m}, y_{t+1}, \boldsymbol{\tau}_{k}\right)$ at $t+1$, then

$$
\begin{aligned}
1+\mathcal{R}_{t+1}^{s} & =\frac{\phi_{k}^{s}+\varepsilon_{k}^{s *}}{\phi_{j}^{s}} \frac{y_{t+1}}{y_{t}}, \\
1+\pi_{t+1} & =\frac{Z_{j}}{Z_{k}} \frac{y_{t}}{y_{t+1}} \mu_{j} .
\end{aligned}
$$

So far we have implicitly assumed that $A^{s}$, i.e., all outstanding equity shares of class $s$, are actively traded every day. In actual markets, however, a fraction of the outstanding equity shares are seldom traded (stocks held in $401(\mathrm{k})$ accounts, for example). Our theory remains unchanged if we replace $A^{s}$ with $\kappa A^{s}$ for some $\kappa \in[0,1]$ that represents the proportion of the universe of outstanding stocks that are actively traded, and think of the remaining $(1-\kappa) A^{s}$ as being held by nontraders outside the model. In an equilibrium in which dealers do not hold assets (as is the 
case in this section), trade volume for asset class $s$ at date $t$ is $\mathcal{V}_{t}^{s}=2 \alpha^{s} G\left(\varepsilon_{t}^{s *}\right) \kappa A^{s}$. A conventional measure of trade volume is the turnover rate used in the empirical work of Section IVA. According to the theory, the turnover rate on date $t$ is

$$
\mathcal{T}_{t}^{s}=\mathcal{V}_{t}^{s} / A^{s}=2 \alpha^{s} G\left(\varepsilon_{t}^{s *}\right) \kappa
$$

Naturally, a nonzero fraction of inactive stocks (i.e., $\kappa<1$ ) lowers the measured level of the turnover rate. ${ }^{42}$ In a recursive equilibrium, suppose the state at date $t$ is $\mathbf{x}_{t}=\left(A_{t}^{m}, y_{t}, \tau_{j}\right)$, then the turnover rate can be written as $\mathcal{T}_{j}^{s}=2 \alpha^{s} G\left(\varepsilon_{j}^{s *}\right) \kappa$. In the theory as in our empirical work, whenever we use an average, e.g., of equity returns or turnover rates across a set of stocks, we use the arithmetic average, e.g., $\mathcal{R}_{t}^{I}=(1 / N) \sum_{s \in \mathbb{N}} \mathcal{R}_{t}^{s}$ and $\mathcal{T}_{t}^{I}=(1 / N) \sum_{s \in \mathbb{N}} \mathcal{T}_{t}^{s}$ are the average return and the average turnover rate for the universe of stocks in the theory.

The (net) nominal rate on the government bond in state $\mathbf{x}_{t}=\left(A_{t}^{m}, y_{t}, \boldsymbol{\tau}_{j}\right)$ is $q\left(\mathbf{x}_{t}\right)^{-1}-1=\max \left(\omega_{j} / \lambda_{j}^{b}, 1\right)-1 \equiv i_{j}$. Then (22) and (23) imply the Fisher equation (the generalization of (14))

$$
1=\sum_{k \in \mathcal{M}} \sigma_{j k} \frac{1+i_{k}}{\left(1+r_{j}\right)\left(1+\bar{\pi}_{j k}\right)},
$$

where $\bar{\pi}_{j k} \equiv \mu_{j} Z_{j} /\left(\bar{\gamma} Z_{k}\right)-1$ is the average inflation rate between state $\mathbf{x}_{t}$ $=\left(A_{t}^{m}, y_{t}, \boldsymbol{\tau}_{j}\right)$ and state $\mathbf{x}_{t+1}=\left(\mu_{j} A_{t}^{m}, y_{t+1}, \boldsymbol{\tau}_{k}\right)$.

\section{B. Calibration}

We think of one model period as being one day. We set $\theta=1$ in our baseline and abstract from micro-level pricing frictions induced by bargaining. The dividend growth rate is independently log-normally distributed over time, with mean 0.04 and standard deviation 0.12 per annum (e.g., as documented in Lettau and Ludvigson 2005, Table 1). That is, $y_{t+1}=e^{x_{t+1}} y_{t}$, with $x_{t+1} \sim \mathcal{N}\left(\bar{\gamma}-1, \Sigma^{2}\right)$, where $\bar{\gamma}-1=E\left(\log y_{t+1}-\log y_{t}\right)=0.04 / 365$ and $\Sigma=\operatorname{std}\left(\log y_{t+1}-\log y_{t}\right)=0.12 / \sqrt{365}$. The parameter $\delta$ can be taken as a proxy of the riskiness of stocks; a relatively low value ensures the monetary equilibrium exists even at relatively high inflation rates. We choose $\delta=(0.7)^{1 / 365}$, i.e., a productive unit has a 70 percent probability of remaining productive each year. The number of outstanding shares of stocks of every class is normalized to 1 , i.e., $A^{s}=1$ for all $s \in \mathbb{N}$. We set $N=20$ so the number of asset classes in the theory matches the number of synthetic liquidity portfolios considered in the cross-sectional analysis of Section IVC.

We normalize $\alpha^{20}=1$ and calibrate $\left\{\alpha^{s}\right\}_{s=1}^{19}$ so that the long-run time-average (under the invariant distribution of monetary policy shocks) of the equilibrium

\footnotetext{
${ }^{42}$ The Turnover column in Table 2 reports the annual turnover rates corresponding to each of the 20 portfolios we studied in Section IVC. Notice that the turnover rates appear to be quite low: even the top 5 percent most traded stocks are only traded about 3 times per year, on average, which suggests that the model should allow for the possibility of $\kappa<1$. Clearly, differences in $\kappa$ across assets would imply differences in turnover across assets. We assume $\kappa$ is the same across assets and focus on differences in turnover generated by heterogeneity in $\alpha^{s}$ because, since $\kappa$ does not enter the asset pricing equations, differences in $\kappa$ are not helpful to understand the facts documented in Section IV.
} 
turnover rate of portfolio $s \in\{1, \ldots, 19\}$ relative to portfolio 20 (i.e., $\overline{\mathcal{T}}^{s} / \overline{\mathcal{T}}^{20}$, where $\left.\overline{\mathcal{T}}^{s} \equiv \lim _{T \rightarrow \infty}(1 / T) \sum_{t=1}^{T} \mathcal{T}_{t}^{S}\right)$ matches the ratio of the average turnover rate of the sth and the twentieth synthetic liquidity portfolio in our sample. Idiosyncratic valuation shocks are drawn from a log-normal distribution. The parameters of the log-normal and the fraction of actively traded stocks, $\kappa$, are chosen so that under the baseline monetary policy process, in response to an unexpected innovation to the policy rate, the theory generates: (i) a marketwide stock return (i.e., $\mathcal{R}_{t}^{I}$ ) on the day of the policy change that matches the corresponding empirical HFIV estimate documented in Table 1, and (ii) a change in the marketwide turnover rate (i.e., $\mathcal{T}_{t}^{I}$ ) on the fifth day after the policy change that matches the corresponding empirical estimate from the VAR in Section IVD. ${ }^{43}$

We estimate the stochastic process for the nominal policy rate, $\left\{\hat{l}_{t}\right\}_{t=0}^{\infty}$, using data for the rate on the 3-month Eurodollar future contract. We formulate that the logarithm of the policy rate follows an $\operatorname{AR}(1)$ process, we estimate this process at a daily frequency for every trading day between January 3, 1994 and December 31, 2007, and approximate it with a 7-state Markov chain, $\left\{\hat{l}_{j},\left[\hat{\sigma}_{j k}\right]\right\}_{j, k=1}^{7} \cdot{ }^{44}$ We then use this estimated policy process to calibrate the theoretical monetary policy process, $\left\langle\left(\omega_{j}, \mu_{j}, r_{j}\right),\left[\sigma_{j k}\right]\right\rangle_{j, k \in \mathcal{M}}$, as follows. We set $\left[\sigma_{j k}\right]=\left[\hat{\sigma}_{j k}\right]$, and choose the process of open-market operations, $\left\{\omega_{j}\right\}_{j \in \mathcal{M}}$, that implements an aggregate real value of money that is constant across states, i.e., $Z_{j}=Z$ for all $j \in \mathcal{M} .^{45}$ Then (26) implies $\iota_{k} \approx r_{k}+\pi_{k}$ for all $k \in \mathcal{M}$, where $\iota_{k} \equiv \sum_{j \in \mathcal{M}} \sigma_{k j}\left(1+i_{j}\right)$ is the expected one-period-ahead nominal rate conditional on the current state $\mathbf{x}_{t}=\left(A_{t}^{m}, y_{t}, \boldsymbol{\tau}_{k}\right)$, and $\pi_{k} \equiv \mu_{k} / \bar{\gamma}-1$ is the average inflation rate between state $\mathbf{x}_{t}=\left(A_{t}^{m}, y_{t}, \boldsymbol{\tau}_{k}\right)$ and any state $\mathbf{x}_{t+1}$. Let $\bar{l}$, $\bar{r}$, and $\bar{\pi}$, denote the empirical means of the nominal policy rate, the real interest rate, and the inflation rate, respectively. Over the sample period 1994-2007, the average nominal policy rate was 0.0447 and the average inflation rate was 0.0269 , so (14) implies a real rate of 0.0178 per annum. ${ }^{46}$ Hence, $1+\bar{\imath}=(1.0447)^{1 / 365}$,

\footnotetext{
${ }^{43}$ This procedure delivers $\kappa=0.016, \quad \ln \varepsilon_{t} \sim \mathcal{N}(-0.2332,1.5705), \quad \alpha^{1}=0.1218, \quad \alpha^{2}=0.1707$, $\alpha^{3}=0.1972, \quad \alpha^{4}=0.2224, \quad \alpha^{5}=0.2438, \quad \alpha^{6}=0.2590, \quad \alpha^{7}=0.2748, \quad \alpha^{8}=0.2939, \quad \alpha^{9}=0.3121$, $\alpha^{10}=0.3306, \alpha^{11}=0.3492, \alpha^{12}=0.3679, \alpha^{13}=0.3899, \alpha^{14}=0.4149, \alpha^{15}=0.4445, \alpha^{16}=0.4821$ $\alpha^{17}=0.5284, \alpha^{18}=0.6011, \alpha^{19}=0.7151$.

${ }^{44}$ Specifically, the process we estimate is $\ln i_{t}=(1-\xi) \ln i_{0}+\xi \ln i_{t-1}+\epsilon_{t}$, where $\epsilon_{t}$ is Gaussian white noise. With $i_{t}$ denominated in bps, the estimates are $\xi=0.9996695, E\left(\ln i_{t}\right)=\ln i_{0}=5.990701$, and $\sqrt{E\left(\epsilon_{t}^{2}\right)}=0.0114289$. Hence, the estimated mean and standard deviation of the nominal rate, $i_{t}$, are $E\left(i_{t}\right)=441$ and $\sqrt{\operatorname{var}\left(i_{t}\right)}=\sqrt{E\left(\epsilon_{t}^{2}\right) /\left(1-\xi^{2}\right)}=206.2516$. The estimated AR(1) process is very persistent so, as suggested by Galindev and Lkhagvasuren (2010), we use the Rouwenhorst method to compute the approximating Markov matrix and states. The code for the Rouwenhorst method is also from Galindev and Lkhagvasuren (2010).

${ }^{45}$ The precise process of open-market operations is described in online Appendix Section C (Proposition 12). This policy implies the real price of money does not change at the times when monetary policy switches states. Consider a state $\mathbf{x}_{t}=\left(A_{t}^{m}, y_{t}, \boldsymbol{\tau}_{i}\right)$. The relevant nominal prices in the model are the dollar price of the second-subperiod consumption good, $1 / \phi^{m}\left(\mathbf{x}_{t}\right)=A_{t}^{m} /\left(Z_{i} y_{t}\right)$, and the dollar price of an equity share, $p^{s}\left(\mathbf{x}_{t}\right)=A_{t}^{m}\left(\varepsilon_{i}^{s *}+\phi_{i}^{s}\right) y_{t} /\left(Z_{i} y_{t}\right)$. Under the policy $\left\{\omega_{j}\right\}_{j \in \mathcal{M}}$ that implements $Z_{j}=Z$ for all $j \in \mathcal{M}, \phi^{m}\left(\mathbf{x}_{t}\right)$ is invariant to monetary policy surprises on impact, and $p^{s}\left(\mathbf{x}_{t}\right)$ responds only if the policy surprise has an effect on the real cum-divided equity price. Thus, this process of open-market operations makes our flexible-price model consistent with the fact that nominal prices in the data typically do not jump when there is a surprise change in the nominal policy rate, even when the policy shock may imply a change in the path of expected inflation.

${ }^{46} \mathrm{As}$ in Section IVA for the policy rate we use the 3-month Eurodollar futures rate (series IEDCS00 produced by the CME Group available via Datastream). The annual average inflation rate is imputed as $[\text { CPI(January_2008)/CPI(January_1994) }]^{1 / 14}-1$, where CPI(Month_Year) is monthly CPI index available from FRED at https://fred.stlouisfed.org/series/CPIAUCSL.
} 
$1+\bar{r}=(1.0178)^{1 / 365}$, and $1+\bar{\pi}=(1.0269)^{1 / 365}$. For each $k \in \mathcal{M}$, we set $r_{k}=\bar{r}+w \hat{\eta}_{k}$ and $\pi_{k} \equiv \bar{\pi}+(1-w) \hat{\eta}_{k}$, where $\hat{\eta}_{k} \equiv \hat{\imath}_{k}-\bar{l}$, and $w \in[0,1]$ indexes the degree of pass-through from nominal rates to real rates. We use $w=0.8$ as baseline, which implies a $100 \mathrm{bp}$ increase in the nominal rate is associated with a $80 \mathrm{bp}$ increase in the real rate and a $20 \mathrm{bp}$ increase in expected inflation. ${ }^{47}$

\section{Simulation}

In this section we conduct two experiments to assess the ability of the theory to match the evidence documented in Section IV. In both experiments, we simulate the calibrated model as follows. First, compute the equilibrium functions characterized by (21)-(25). Second, simulate 1,000 samples of the dividend, each of length equal to our data sample. Then set the path of the nominal rate in the model equal to the actual empirical path of the policy rate used in our empirical work. Finally, compute the equilibrium path of the model 1,000 times (one for each realization of the simulated dividend path), and for each simulated equilibrium path, compute the average daily equity return for each asset class.

Experiment 1: Disaggregative Announcement-Day Effects.-The first experiment is the model analogue of the cross-sectional analysis of Section IVB. For each of the 20 asset classes, we run an event-study regression for announcement-day returns 1,000 times (one for each of the 1,000 simulated equilibrium paths for daily stock return for that particular asset class). The results are illustrated in Figure 6, which reports the empirical HFIV estimates from Table 2 along with the regression estimates from the simulated model. ${ }^{48}$ For each theoretical portfolio, the value displayed in Figure 6 is the average E-based estimate over the model 1,000 simulations. The 99 percent confidence intervals for the theoretical estimates are constructed using the distribution of estimates from the 1,000 model simulations. The 99 percent confidence intervals for the empirical estimates are from the HFIV regressions from Section IVC. The model was calibrated so that the marketwide response to the policy shock on the announcement day matches the empirical HFIV estimate of Table 1. We are interested in whether the theory can account for the profile of returns across stocks with different turnover liquidity, the hallmark of the turnover-liquidity transmission mechanism. Figure 6 shows the theory is able to generate most of the announcement-day tilting in cross-sectional returns. The fit is excellent for the first 14 liquidity portfolios. For the 6 most liquid portfolios, the model predicts a bit less tilting than the data.

Experiment 2: Impulse Responses.-The second experiment is the model analogue of the VAR analysis of Section IVD. Figure 7 reports the model-generated

\footnotetext{
${ }^{47}$ This choice is guided by the pass-through estimates in Gertler and Karadi (2015) at a two-year horizon. As a robustness check, we have also set $w=1$ and recalibrated the model to fit the same data targets as the baseline calibration, and found that the quantitative performance of the theory is very similar to the case with $w=0.8$. In online Appendix Section D.D6 we report results for the case with $w=0$.

${ }^{48}$ Since the monetary policy is exogenous in the model, the E-based estimates based on the synthetic data are not subject to the biases discussed in Section IVB. For this reason, here we use the HFIV empirical estimates as a benchmark for comparison.
} 


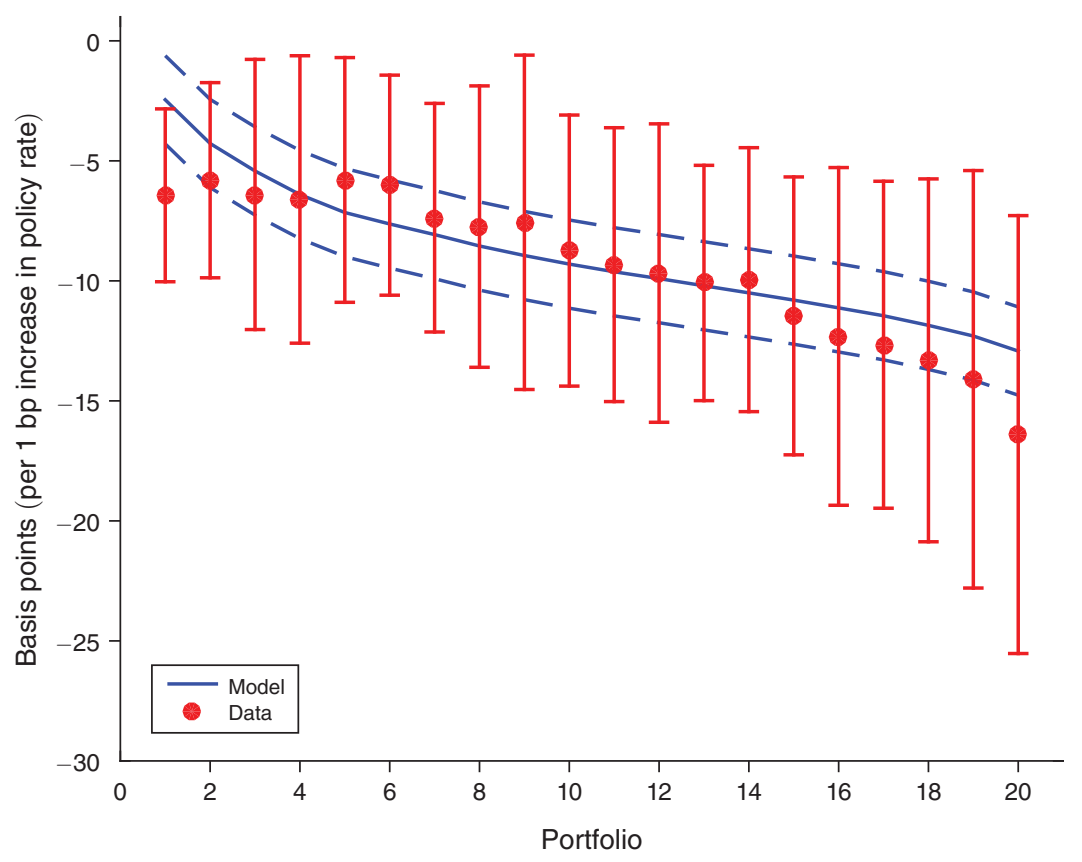

Figure 6. Cross-Sectional Announcement-Day Responses of Stock Returns to Policy Rate

impulse responses for the policy rate, the cumulative marketwide stock return, and the average turnover rate to a $1 \mathrm{bp}$ increase in the policy rate, along with the corresponding empirical impulse responses and 99 percent confidence intervals estimated from actual data (those described in Section IVD). The top and bottom panels show responses for forecast horizons of 30 days and 120 days, respectively. The path of the policy rate from the model is quite close to the empirical path. The middle panels show the response of the daily cumulative stock return. On impact, in response to the $1 \mathrm{bp}$ unexpected increase in the nominal rate, the model stock return falls by 9.38 bps: the same as the HFIV estimate of Table 1, as targeted by the calibration. Since persistence (of the policy shock and turnover liquidity) is an essential element of the theoretical mechanism, we are interested in whether the model can account for the dynamics of the response of the cumulative return. The theoretical and empirical impulse responses for subsequent days after the policy shock remain quite close. For example, the theoretical impulse response can account for over 80 percent of the empirical response for the first 30 days, and for at least 66 percent of the empirical response for the subsequent 90 days. ${ }^{49}$

The right panels of Figure 7 show the response of the level of the daily turnover rate. The model was calibrated so that the response of turnover on day 5 after the announcement matches the empirical estimate. On impact, in response to a $1 \mathrm{bp}$ unexpected increase in the nominal rate, the turnover rate falls by $-6.8502 \times 10^{-6}$ in the model. The model response for turnover is about seven times smaller than the

\footnotetext{
${ }^{49}$ The simulated theoretical cumulative return is $-8.7 \mathrm{bp}$ on day 30 , and $-7.7 \mathrm{bp}$ on day 120 . The estimated empirical cumulative return is $-11.0 \mathrm{bp}$ on day 30 , and -11.7 bp on day 120 .
} 

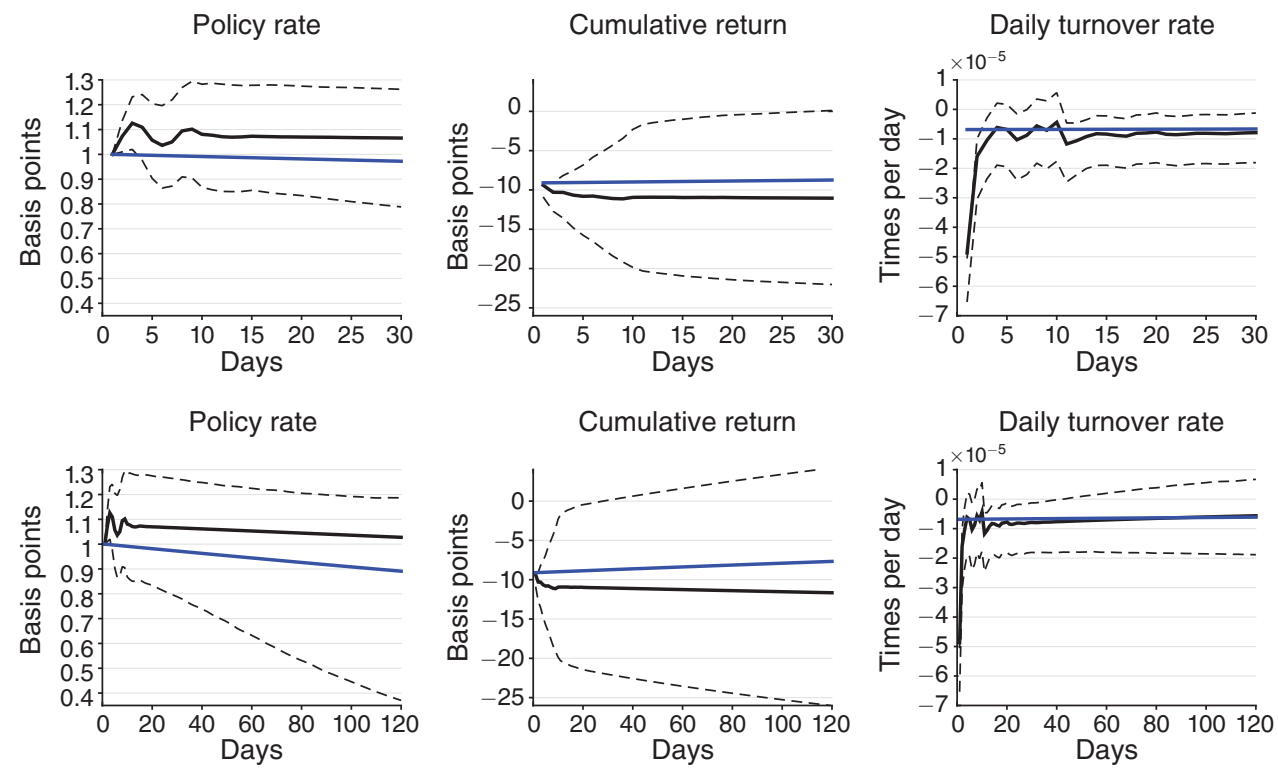

Figure 7. Empirical and Theoretical Impulse Responses to a 1 Basis Point Increase in the Policy Rate

Notes: The darker solid line is the estimated empirical impulse response. Broken lines are the 95 percent confidence bands of the empirical impulse response.

empirical estimate $\left(-4.8692 \times 10^{-5}\right.$ according to the empirical impulse response) . However, although the model response for turnover is much smaller on impact, it is very persistent and remains relatively close to the empirical response at longer horizons. For example, the difference between the empirical path for the turnover rate and the theoretical path becomes statistically insignificant for all days after day 3. Both the empirical and the theoretical responses are quite persistent. This persistent effect of policy on the turnover rate is consistent with a response in return that is quantitatively in line with the data, even though the announcement-day effect on turnover is much smaller than in the data.

\section{Conclusion}

We conclude by mentioning what we think are three promising avenues for future work. First, in the model we have presented, all asset purchases are paid for with outside money. In other words, the theory focuses on the relevant margin for settings, transactions, or traders for which credit limits have become binding. While arguably stark, we think this formulation is a useful benchmark to contrast with the traditional asset-pricing literature that abstracts from the role of costly or scarce payment instruments. Having said this, we think it would be useful to extend the theory to allow for credit arrangements. The possibility of buying on margin, for example, is likely to interact with the monetary mechanisms we have emphasized here in interesting ways (see Lagos and Zhang 2018 for work along these lines). Second, given that trading frictions in the exchange process are at the center of the analysis (e.g., the likelihood of finding a counterparty, or the market power of dealers who 
intermediate transactions), it would be interesting to endogenize them (see Lagos and Zhang 2015 for work in this direction). Third, while we have focused on stocks in our empirical work, the transmission mechanism we have identified is likely to be operative, and possibly even more conspicuous, in markets for other assets, such as Treasury securities and assets that trade in more frictional over-the-counter markets.

\section{REFERENCES}

Ang, Andrew, Assaf A. Shtauber, and Paul C. Tetlock. 2013. "Asset Pricing in the Dark: The Cross-Section of OTC Stocks.” Review of Financial Studies 26 (12): 2985-3028.

Bernanke, Ben S., and Kenneth N. Kuttner. 2005. "What Explains the Stock Market's Reaction to Federal Reserve Policy?" Journal of Finance 60 (3): 1221-57.

Cochrane, John H., and Monika Piazzesi. 2002. "The Fed and Interest Rates: A High-Frequency Identification.” American Economic Review 92 (2): 90-95.

Cook, Timothy, and Thomas Hahn. 1989. "The Effect of Changes in the Federal Funds Rate Target on Market Interest Rates in the 1970s.” Journal of Monetary Economics 24 (3): 331-51.

Duffie, Darrell, Nicolae Gârleanu, and Lasse Heje Pedersen. 2005. "Over-the-Counter Markets." Econometrica 73 (6): 1815-47.

Duffie, Darrell, Nicolae Gârleanu, and Lasse Heje Pedersen. 2007. "Valuation in Over-the-Counter Markets." Review of Financial Studies 20 (6): 1865-1900.

Fama, Eugene F., and Kenneth R. French. 1988. "Permanent and Temporary Components of Stock Prices." Journal of Political Economy 96 (2): 246-73.

-Galindev, Ragchaasuren, and Damba Lkhagvasuren. 2010. "Discretization of Highly Persistent Correlated AR(1) Shocks.” Journal of Economic Dynamics and Control 34 (7): 1260-76.

Gârleanu, Nicolae. 2009. "Portfolio Choice and Pricing in Illiquid Markets." Journal of Economic Theory 144 (2): 532-64.

-Gertler, Mark, and Peter Karadi. 2015. "Monetary Policy Surprises, Credit Costs, and Economic Activity." American Economic Journal: Macroeconomics 7 (1): 44-76.

Gonçalves, Sílvia, and Lutz Kilian. 2004. "Bootstrapping Autoregressions with Conditional Heteroskedasticity of Unknown Form.” Journal of Econometrics 123 (1): 89-120.

Gorodnichenko, Yuriy, and Michael Weber. 2016. "Are Sticky Prices Costly? Evidence from the Stock Market." American Economic Review 106 (1): 165-99.

Gürkaynak, Refet S., Brian P. Sack, and Eric T. Swanson. 2007. "Market-Based Measures of Monetary Policy Expectations." Journal of Business and Economic Statistics 25 (2): 201-12.

-Hamilton, James D. 2003. "What Is an Oil Shock?” Journal of Econometrics 113 (2): 363-98.

- Harrison, J. Michael, and David M. Kreps. 1978. "Speculative Investor Behavior in a Stock Market with Heterogeneous Expectations." Quarterly Journal of Economics 92 (2): 323-36.

Kilian, Lutz. 2008a. "The Economic Effects of Energy Price Shocks." Journal of Economic Literature 46 (4): 871-909.

Kilian, Lutz. 2008b. "Exogenous Oil Supply Shocks: How Big Are They and How Much Do They Matter for the US Economy?" Review of Economics and Statistics 90 (2): 216-40.

Kuttner, Kenneth N. 2001. "Monetary Policy Surprises and Interest Rates: Evidence from the Fed Funds Futures Market." Journal of Monetary Economics 47 (3): 523-44.

Lagos, Ricardo, and Guillaume Rocheteau. 2009. "Liquidity in Asset Markets with Search Frictions." Econometrica 77 (2): 403-26.

Lagos, Ricardo, Guillaume Rocheteau, and Pierre-Olivier Weill. 2011. "Crises and Liquidity in Overthe-Counter Markets.” Journal of Economic Theory 146 (6): 2169-2205.

Lagos, Ricardo, and Shengxing Zhang. 2015. "Monetary Exchange in Over-the-Counter Markets: A Theory of Speculative Bubbles, the Fed Model, and Self-Fulfilling Liquidity Crises." NBER Working Paper 21528.

Lagos, Ricardo, and Shengxing Zhang. 2018. "On Money as a Medium of Exchange in Near-Cashless Credit Economies." Unpublished.

Lettau, Martin, and Sydney C. Ludvigson. 2005. "Expected Returns and Expected Dividend Growth.” Journal of Financial Economics 76 (3): 583-626.

Mertens, Karel, and Morten O. Ravn. 2013. "The Dynamic Effects of Personal and Corporate Income Tax Changes in the United States." American Economic Review 103 (4): 1212-47.

Rigobon, Roberto, and Brian Sack. 2004. "The Impact of Monetary Policy on Asset Prices." Journal of Monetary Economics 51 (8): 1553-75. 
Romer, Christina D., and David H. Romer. 1989. "Does Monetary Policy Matter? A New Test in the Spirit of Friedman and Schwartz." In NBER Macroeconomics Annual: 1989, Vol. 4, edited by Olivier Jean Blanchard and Stanley Fischer, 121-84. Cambridge, MA: MIT Press.

Stock, James H., and Mark W. Watson. 2012. "Disentangling the Channels of the 2007-2009 Recession.” NBER Working Paper 18094.

Thorbecke, Willem. 1997. "On Stock Market Returns and Monetary Policy.” Journal of Finance 52 (2): 635-54.

-Vayanos, Dimitri, and Pierre-Olivier Weill. 2008. "A Search-Based Theory of the On-the-Run Phenomenon.” Journal of Finance 63 (3): 1361-98.

-Weill, Pierre-Olivier. 2007. "Leaning against the Wind." Review of Economic Studies 74 (4): 1329-54. 